BMC

Genomics

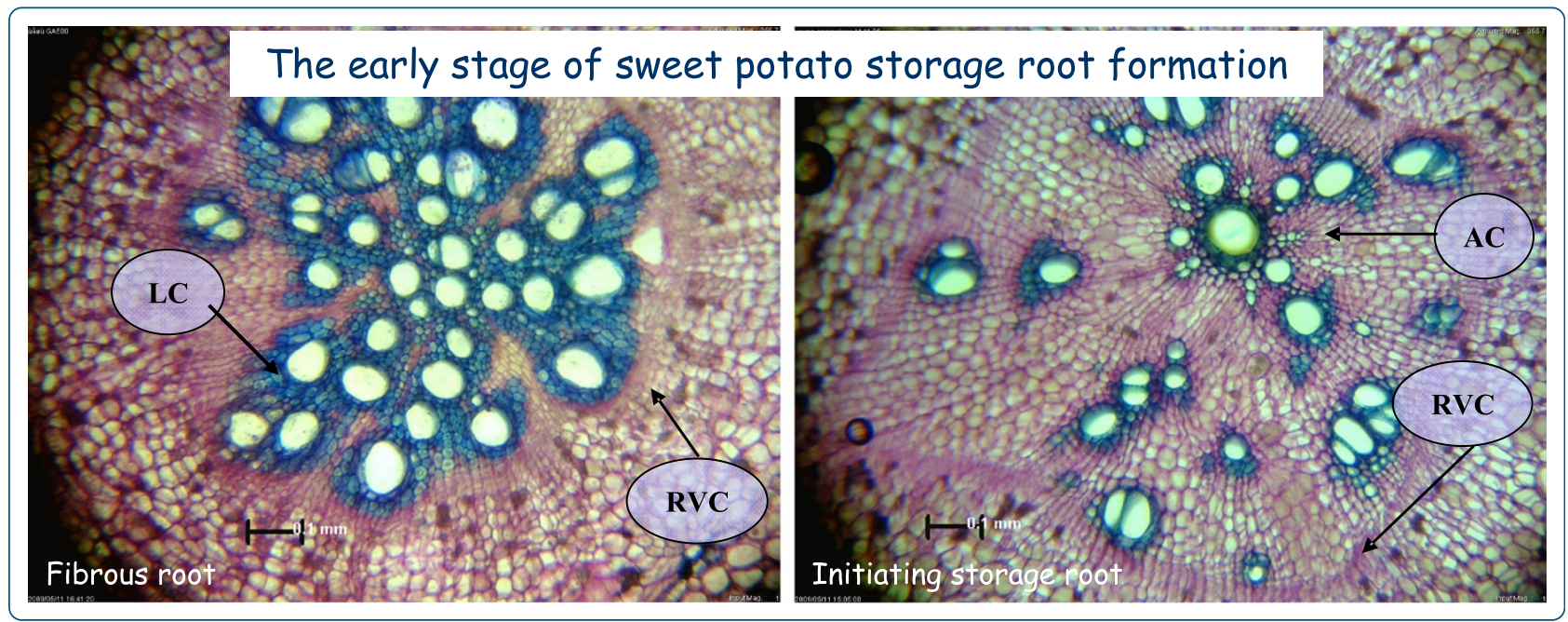

Transcriptional profiling of sweetpotato (Ipomoea batatas) roots indicates down-regulation of lignin biosynthesis and up-regulation of starch biosynthesis at an early stage of storage root formation

Firon et al. 


\title{
Transcriptional profiling of sweetpotato (Ipomoea batatas) roots indicates down-regulation of lignin biosynthesis and up-regulation of starch biosynthesis at an early stage of storage root formation
}

Nurit Firon ${ }^{1 *}$, Don LaBonte ${ }^{2}$, Arthur Villordon ${ }^{3}$, Yanir Kfir ${ }^{1}$, Julio Solis², Evgenia Lapis ${ }^{1}$, Temima Schnitzer Perlman ${ }^{4}$, Adi Doron-Faigenboim ${ }^{1}$, Amots Hetzroni ${ }^{5}$, Leviah Althan ${ }^{1}$ and Lahan Adani Nadir ${ }^{1}$

\begin{abstract}
Background: The number of fibrous roots that develop into storage roots determines sweetpotato yield. The aim of the present study was to identify the molecular mechanisms involved in the initiation of storage root formation, by performing a detailed transcriptomic analysis of initiating storage roots using next-generation sequencing platforms. A two-step approach was undertaken: (1) generating a database for the sweetpotato root transcriptome using 454-Roche sequencing of a cDNA library created from pooled samples of two root types: fibrous and initiating storage roots; (2) comparing the expression profiles of initiating storage roots and fibrous roots, using the Illumina Genome Analyzer to sequence cDNA libraries of the two root types and map the data onto the root transcriptome database.

Results: Use of the 454-Roche platform generated a total of 524,607 reads, $85.6 \%$ of which were clustered into 55,296 contigs that matched 40,278 known genes. The reads, generated by the Illumina Genome Analyzer, were found to map to 31,284 contigs out of the 55,296 contigs serving as the database. A total of 8,353 contigs were found to exhibit differential expression between the two root types (at least 2.5 -fold change). The Illumina-based differential expression results were validated for nine putative genes using quantitative real-time PCR. The differential expression profiles indicated down-regulation of classical root functions, such as transport, as well as down-regulation of lignin biosynthesis in initiating storage roots, and up-regulation of carbohydrate metabolism and starch biosynthesis. In addition, data indicated delicate control of regulators of meristematic tissue identity and maintenance, associated with the initiation of storage root formation.
\end{abstract}

Conclusions: This study adds a valuable resource of sweetpotato root transcript sequences to available data, facilitating the identification of genes of interest. This resource enabled us to identify genes that are involved in the earliest stage of storage root formation, highlighting the reduction in carbon flow toward phenylpropanoid biosynthesis and its delivery into carbohydrate metabolism and starch biosynthesis, as major events involved in storage root initiation. The novel transcripts related to storage root initiation identified in this study provide a starting point for further investigation into the molecular mechanisms underlying this process.

Keywords: Fibrous root, Ipomoea batatas, Lignin biosynthesis, Starch biosynthesis, Storage-root initiation, Transcription profiling

\footnotetext{
*Correspondence: vcfiron@volcani.agri.gov.il

${ }^{1}$ Institute of Plant Sciences, The Volcani Center, Agricultural Research

Organization, Bet Dagan 50250, Israel

Full list of author information is available at the end of the article
} 


\section{Background}

Sweetpotato [Ipomoea batatas (L.) Lam.] is the seventh most important food crop in the world [1]. It has a worldwide production of approximately 107 million metric tons and is the third most important root/tuber crop after potato (Solanum tuberosum L.) and cassava (Manihot esculenta Crantz). World production is centered in Southeast Asia, with China being the largest producer, while Sub-Saharan Africa ranks second [2]. Sweetpotato is used as a source of starch, ethanol, and animal fodder in most of Asia while it is considered a subsistence crop in Africa. The USA, Japan, Australia, New Zealand, South Africa and Israel are among the few producing countries that grow sweetpotato as a vegetable to market in developed economies. Global demand for sweetpotato is on the rise due to its high nutritional value. The Nutrition Action Health Letter from the Center for Science in the Public Interest, USA [3], rates sweetpotatoes as the highest ranking vegetable for human nutrition.

The most economically important physiological process in sweetpotato production is storage root (SR) development. Sweetpotatoes are commercially propagated through vegetative cuttings. These cuttings produce adventitious roots that give rise to the SRs [4-7]. Adventitious roots originate from primordia located on the nodes, as well as from the cut ends, i.e., "wound" roots [4,6]. Initially, white fibrous roots (FRs) develop and some of these subsequently develop into SRs. Depending on the number of FRs induced to form SRs, sweetpotato plants will yield either a high (4 to 8 per plant) or low number of SRs that may even be reduced to one very large SR per plant [7]. Togari [4] described the sequence of anatomical events leading to SR initiation in varieties Okinawan and Beinakzi and reported that the regular vascular cambium layer first appears 20 days after transplanting (DAT), followed by the initial development of secondary anomalous cambium (AC) features at 25 DAT. Togari [4] also documented the incidence of stele lignification and proposed that lignification prevents SR initiation. Wilson and Lowe [5] also suggested that only the appearance of $\mathrm{AC}$ can prevent stele lignification.

Recently, we demonstrated in both Georgia Jet and Beauregard sweetpotato varieties (the leading Israeli variety and an important USA variety, respectively), that the period spanning 5 to 35 DAT is critical in determining whether adventitious roots become lignified or initiate as SRs, and that the appearance of AC marks the initial phase of SR formation [7]. The molecular mechanisms underlying the induction of adventitious roots to become SRs are, however, poorly understood. Expression studies have been used in an effort to elucidate factors involved in SR formation. You et al. [8] constructed a cDNA library of early-stage SRs, and identified 22 genes differentially expressed between FRs (non-storage and storage stage) and SRs. Among them were a no apical meristem (NAM)like and a MADS-box (MCM1, AGAMOUS, DEFICIENS and SRF) protein gene, both of which were down-regulated in SRs. McGregor [9] found several NAC family transcriptional regulator proteins that were down regulated in storage roots, similar to the NAM-like protein described by You et al. [8]. McGregor [9] also identified up-regulated expression of two NAM-like genes, as well as sporamin genes and genes involved in starch biosynthesis, in storage roots that developed six weeks after planting compared to fibrous roots. Several additional MADS-box genes (IbMADS1, IbMADS3, IbMADS4, IbMADS10, IbMADS79, IbAGL17, IbAGL2O and SRD1) expressed in root tissues have been isolated from sweetpotato, and their possible roles in root development have been deduced [10-15]. Tanaka et al. [16] identified 10 genes with differential expression among FRs, thick roots, and SRs. One of the genes, SRF6, encoded a receptor-like kinase with high expression around the primary cambium and xylem meristem. In addition, Tanaka et al. [17] suggested three sweetpotato class 1 knotted1-like homeobox (KNOX1) genes as possible regulators of cytokinin levels in SRs.

De-novo assembly of transcript sequences produced by next-generation sequencing (NGS) technologies offers a rapid approach to obtaining expressed gene sequences for non-model organisms. Indeed, most recently, NGS was used by several research groups to obtain leaf, stem and root transcriptome data for different sweetpotato cultivars [18-20]. Tao et al. [20] used Illumina NGS, employing a combination of different tissues at different developmental stages, to generate 51,736 annotated transcripts, and identified differentially expressed transcripts in different tissues, including roots. Xie et al. [21] analyzed the transcriptome of a purple sweetpotato, obtaining a total of 58,800 unigenes, and suggested UDP-glucose-flavonoid 3-O-glucosyltransferase as one of the key enzymes in anthocyanin biosynthesis and that anthocyanin-3-glucoside might be one of the major components for anthocyanin pigments in the purple sweetpotato.

The presently described study focused on the identification of the molecular mechanisms involved in the initiation of SR formation in the leading sweetpotato variety in Israel, Georgia Jet, by performing a detailed transcriptomic analysis of initiating SRs (ISRs) using NGS platforms. A two-step approach was undertaken: (1) generating a database for the sweetpotato root transcriptome using 454-Roche sequencing of a cDNA library created from pooled samples of two root types: FRs and ISRs; (2) comparing the expression profiles of ISRs and FRs, using the Illumina Genome Analyzer to sequence non-normalized cDNA libraries of the two root types and mapping the data onto the root transcriptome database. Use of the 454-Roche platform generated a total of 524,607 reads, $85.6 \%$ of which were clustered into 55,296 contigs that matched 40,278 known 
genes. The differential expression profiles between the two root types obtained by the Illumina platform indicated down-regulation of classical root functions, such as transport and response to the environment, and of lignin biosynthesis in ISRs, along with up-regulation of carbohydrate metabolism and starch biosynthesis. In addition, the data suggest delicate control of stem cell maintenance and differentiation in sweetpotato vascular development associated with the initiation of SR formation.

\section{Results and discussion}

Insight into the transcriptome of sweetpotato roots

Defining the transcriptome using 454 sequencing and de-novo assembly

To obtain insight into the molecular mechanisms involved in the initiation of SR formation in sweetpotato, and to identify candidate genes involved in this process, a two-stage approach was adopted. First, a database of the sweetpotato (var. Georgia Jet) root transcriptome was generated, using 454-Roche sequencing of a cDNA library created from pooled RNA samples of two root types: ISRs and non-initiating FRs. Roots were divided into either ISRs or FRs following microscopic analysis, as shown in Figure 1. Second, the expression profiles of ISRs and FRs were compared, using the Illumina Genome Analyzer, to sequence non-normalized cDNA libraries of the two root types and the data were mapped onto the root transcriptome database.

A total of 524,607 high-quality reads were generated with an average read length of $310 \mathrm{bp}$. The total number of bases (without keys, tags or bad-quality bases) was $1.63 \mathrm{E}+08$. The MIRA assembly clustered $85.6 \%(449,282$ sequences) of the 454 sequence reads into 55,296 contigs. The sequence length distribution is illustrated in Figure 2. The average length of the contigs was $519 \pm 245$ bases. The remaining 75,325 (orphan) sequences were retained as singletons. The clustered contig data are available via a web page connected to The Volcani Center, Agricultural Research Organization web page at http://batata.agri.gov.il/.

\section{Functional annotation by sequence comparison with public databases}

The transcriptomic data were used to query public genomic databases [i.e. NCBI non-redundant $(\mathrm{Nr})$ ] using BLASTX. Of the 55,296 contigs, 40,278 (73\%) matched known genes at a cut-off $\mathrm{E}$ value $\leq 1.0 \mathrm{E}-3$. Annotations of the two best hits for each contig are given at http://batata.agri.gov.il/ and in Additional file 1. E-value distribution for the top BLAST result for each sequence is given in Figure 3. The E-value distribution of the top hits in the $\mathrm{Nr}$ database revealed that $99.5 \%$ of the mapped sequences show significant homology (less than 1.0E-5), and 22\% of the sequences showed greater than $80 \%$ similarity. These results indicated a high level of homology between our sequences and those

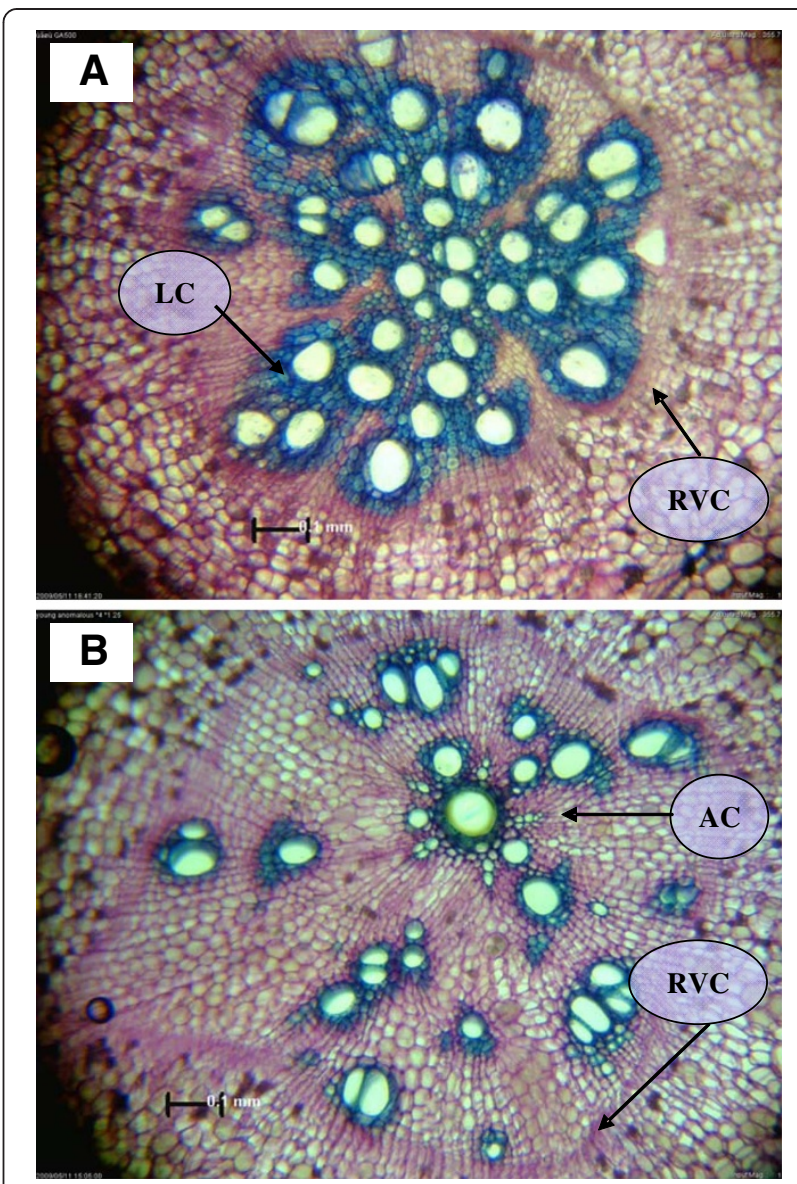

Figure 1 Microscopic view of adventitious root cross sections 26 days after transplanting. A. Fibrous root (FR) cross section. B. Initiated storage root (ISR) cross section. Tissue sections were taken at $3 \mathrm{~cm}$ from the proximal root part and stained with toluidine blue. LC - lignified cells; AC - anomalous cambium; RVC - regular vascular cambium. Scale bars $=0.1 \mathrm{~mm}$.

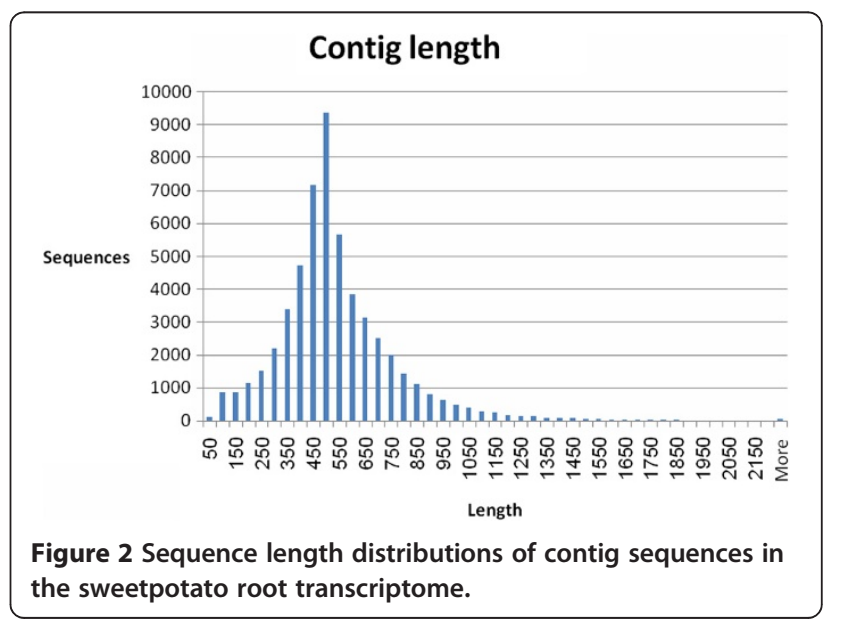




\section{E-value Distribution}

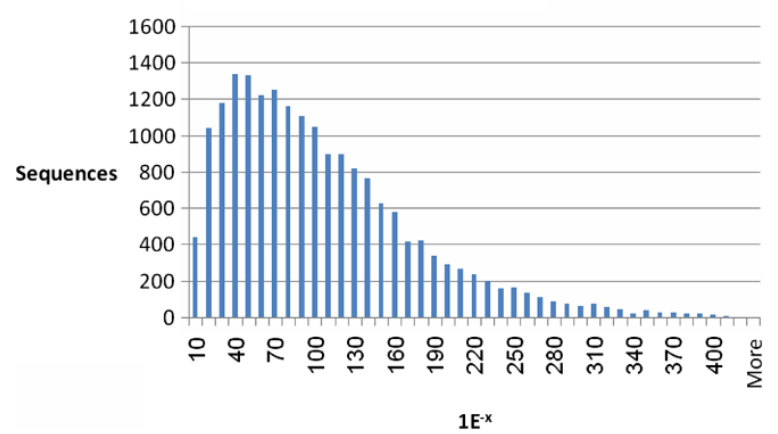

Figure 3 E-value distribution of contig sequences' BLAST results in the sweetpotato root transcriptome.

found in the BLAST database. Similarity distribution of the contigs to their BLAST results is illustrated in Figure 4. Species distribution of the BLAST results is given in Additional file 2, demonstrating that most sweetpotato sequences exhibited similarity to Vitis vinifera, Ricinus communis and Populus trichocarpa sequences (43\%, 24\% and $18 \%$, respectively), as well as to members of the Solanaceae family. Similarity to sequences of Arabidopsis thaliana was less than $10 \%$. The relatively low number of hits detected with Ipomoea batatas (sweetpotato) may be attributed to the low number of publicly available sequences in the database. The sweetpotato root transcript sequences generated in this study thus add to the recently accumulated sweetpotato sequences [18-21] which can be used for the discovery of new genes involved in root development and functioning and in the initiation of SR formation.

\section{Functional classification by gene ontology (GO) and by the} Kyoto encyclopedia of genes and genomes (KEGG)

To assess whether the sweetpotato root transcriptomic data were indeed representative of roots and SRs, the annotated contigs were assigned to molecular functions using GO. BlastoGO [22] was used to obtain BLAST

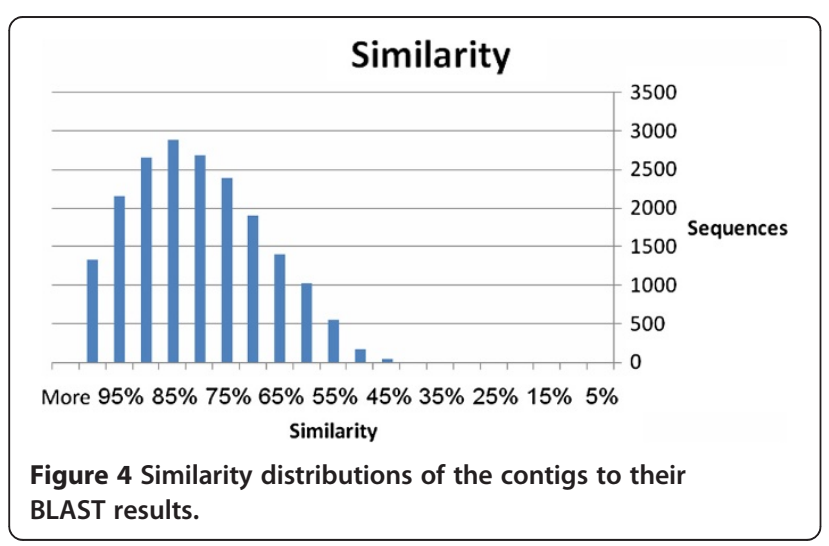

results for our contigs against the NCBI $\mathrm{Nr}$ database and then again to obtain GO annotations for the BLAST results. Ontologizer [23] was used to perform the GO functional classification for the contigs. Of the 40,278 contigs that matched known genes, 34,308 sequences could be grouped into 4,776 different GO categories, and all parental GO terms [i.e. level 2: Biological Process (BP), Molecular Function (MF), Cellular Component (CC)] were assigned (data not shown). Of the GO annotations, $55.3 \%$ were associated with BP, $34.5 \%$ were associated with MF, and 10.2\% were associated with CC (2640, 1648 and 487 , respectively). The contigs were further classified using GOSlim [24] and results are presented in Figure 5 and Additional file 3 . There was a high representation of cellular process, metabolic process, primary metabolic process, biosynthetic process, macromolecular metabolic process, response to stimulus, localization and transport in the parental category BP, high representation of binding (including nucleotide, protein, carbohydrate and lipid binding), catalytic, transporter and transcription regulator activities in the MF parental category, and high representation of cell, intracellular, cytoplasm, organelle and membrane-bound organelle in the CC category. Similar results have been recently demonstrated for sweetpotato [19-21] and other root transcriptomes, including rice [25], wild rice [26] and Avena barbata [27], supporting the quality of our Georgia Jet sweetpotato root transcriptome data.

To assign the detected contigs to biological pathways, the 55,296 contig sequences were compared using BLASTX with an E-value cutoff of $<10 \mathrm{E}-3$ against the KEGG biological pathways database. The contigs were mapped to 140 KEGG pathways, as demonstrated in Additional file 4. Figure 6 summarizes the top 20 represented biological pathways including at least 200 contigs. The highly represented pathways contained starch and sucrose metabolism, purine metabolism, methane metabolism, T-cell receptor signaling pathway, glycolysis, amino sugar and nucleotide sugar metabolism, oxidative phosphorylation, phenylalanine metabolism and phenylpropanoid biosynthesis.

Taken together, the generated database of sweetpotato root transcripts (based upon a mixture of FRs and ISRs) contains genes involved in: (a) classical root functions, such as binding and transport (including transmembrane transport and vesicle-mediated transport) as well as responses to the environment (including response to stimulus), in addition to metabolic processes with high representation of oxidation-reduction processes; (b) metabolic processes and regulation of metabolic processes as well as functions related to development. These results demonstrate the value of the generated transcriptomic data to serve as a database and a reference for continued study aimed at detecting early transcriptome changes in sweetpotato adventitious roots upon initiation of SR formation, as detailed below. 

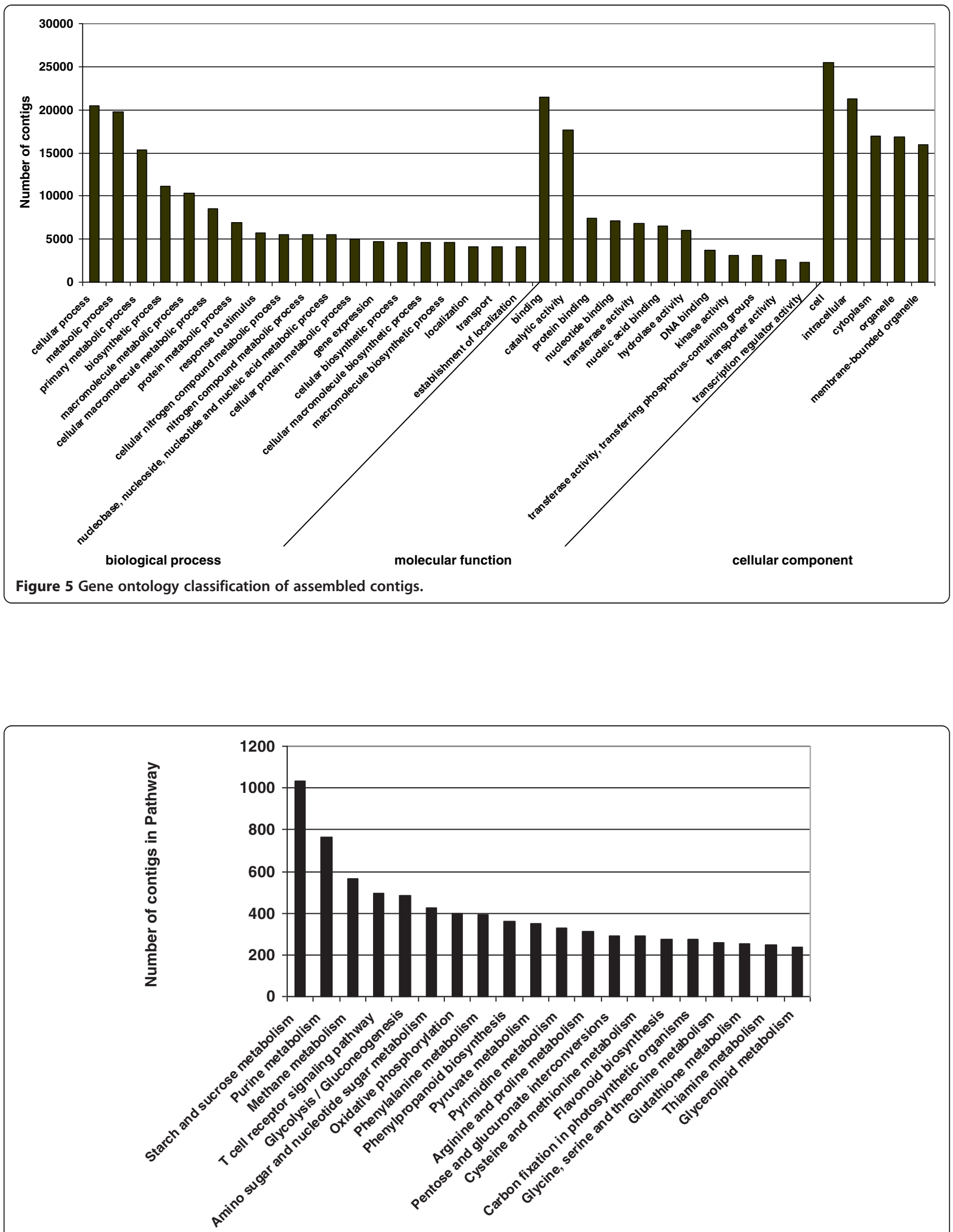

Figure 6 Kyoto Encyclopedia of Genes and Genomes classification of assembled contigs. 


\section{Early transcriptome changes in sweetpotato adventitious roots upon initiation of SR formation}

High-throughput sequencing at the cDNA level, using Illumina GA-IIx technology, was carried out to compare the transcription profiles of two bar-coded samples: (1) a pooled sample of FRs and (2) a pooled sample, of the same age, of ISRs; $17,703,982$ and $14,780,229$ reads ( $1 \times 100$-bp long) were obtained for the FRs and ISRs samples, respectively. The reads were mapped against the set of 55,296 FLX contigs described above, as detailed in 'Methods'. To enable a direct comparison between the two samples, the read count per EST contig was normalized as described in 'Methods'. The results of total normalized read counts in the FR and ISR samples are presented in Additional file 5 and are available via our sweetpotato database http://batata.agri.gov.il/. The reads were found to map to 31,284 contigs out of the 55,296 contigs that served as the database, i.e. 56.6\% (Additional file 5).

A contig was considered differentially expressed between the ISR and FR samples if two conditions were met: (1) at least 10 reads were mapped to this contig in at least one of the samples and (2) no reads were mapped to the contig in the other sample, or the fold change between read number in each sample was at least 2.5. Thus, a total of 8,353 contigs were found to exhibit differential expression between the two samples. Of these, 4,075 (48.8\%) contigs were up-regulated in ISRs compared to FRs and 4,278 (51.2\%) contigs were up-regulated in FRs compared to ISRs (Additional file 6); 803 contigs were found to exhibit at least 10-fold higher read number in ISRs compared to FRs and 1,457 contigs were found to exhibit at least 10-fold higher read number in FRs compared to ISR (including contigs with at least 10 mapped reads in one of the samples and no reads in the other sample; Additional file 6 and http://batata.agri.gov.il/).

\section{Genes involved in sporamin accumulation and carbohydrate metabolism are highly up-regulated during initiation of SR formation}

The list of 70 contigs exhibiting highest differential expression (fold-change) in ISRs compared to FRs is summarized in Table 1. In this list, expression values in the ISR sample, expressed as normalized read number, range between 49 and 4,080 reads per contig and the fold-change in expression compared to the FR sample ranges between 46- and 1,467-fold (Table 1). It should be noted that, for contigs that had no reads in the FR sample, read number was changed from zero to one to enable 'fold-change' calculation (Table 1). Highest fold-change expression levels were obtained for contigs representing various members of the sporamin and $\beta$-amylase genes, exhibiting 174- to 1,467-fold change in expression. The products of these two genes are known to accumulate to high levels in sweetpotato SRs, with sporamin and $\beta$-amylase accounting for about $60 \%$ and $5 \%$ of total soluble proteins in sweetpotato SRs, respectively [28,29]. Up-regulation of a contig representing an expansin gene in the ISR sample was apparent (contig S_PBL_c3870) exhibiting 98 fold-change in expression. Expansins are known as cell wall-loosening proteins affecting cell expansion in plants and were shown to be involved in root development [[30] and references therein]. In the present work, 15 contigs representing expansin gene sequences were detected by Illumina cDNA sequencing (Additional file 5). Of these, 13 exhibited higher expression in ISRs compared with FRs, including 4 contigs (S_PBL_c1016, S_PBL_c6536, S_PBL_c1824, S_PBL_c6257) that showed high read number $(4362,3066,1547$ and 1446 reads, respectively; Table 1). Our data thus suggest the involvement of expansin in initiation of SR formation and contradict previous findings by Noh et al. [31] showing that down-regulation of the IbEXP1 expansin gene (exhibiting high homology to contigs S_PBL_c6536 and S_PBL_c1824) in Ipomoea batatas cv. Yulmi enhanced SR development of sweetpotato.

In addition, included in this list are genes involved in starch biosynthesis, coding for $\alpha$ and $\beta$ subunits of ADP glucose pyrophosphorylase (AGPase; exhibiting high expression levels: 4,080 and 1,394 reads for specific $\beta$ and $\alpha$ members, respectively) and phosphoglucomutase. This list includes a significant number of contigs without annotation (30 contigs; 43\%). Looking into the expression levels (number of Illumina-generated reads) of additional starch biosynthesis genes not included in Table 1, high expression of contigs representing several members of granule-bound starch synthase was detected (in the range of 498 to 4,757 reads, exhibiting 15- to 23-fold higher expression in ISRs compared to FRs, for contigs S_PBL_c3042, S_PBL_c9881, S_PBL_c4145; Additional file 5 and http://batata.agri.gov.il/). The high expression level (83-fold higher in ISR compared to FR) of a contig exhibiting homology to ferredoxin suggests its involvement in the redox modulation and activation of AGPase [32].

To follow changes in starch levels in the roots of Georgia Jet during the first 4 weeks after transplanting, spanning the timing of SR initiation [7], we evaluated starch concentrations in the root system at several time points after transplanting (Figure 7). Samples were taken from the entire root system, since up to 3 weeks after transplanting SR initiation could not be distinguished by microscopic analysis of root cross sections. The data indicated a peak of starch accumulation (an over fivefold increase) at the initiation of SR formation, between 3 and 4 weeks after transplanting (Figure 7). Starch levels of non-initiated, 2-month-old FRs were found to be relatively low ( $0.9 \mathrm{mg} \mathrm{g} \mathrm{fw}^{-1}$; Figure 7), suggesting that the increased starch in the pooled root sample was contributed by the ISRs. These data are in agreement with the elevated 
Table 1 Top 70 up-regulated contigs in initiating storage roots compared to fibrous roots

\begin{tabular}{|c|c|c|c|c|c|}
\hline Contig & Length & ISR reads & FR reads & Fold change & Annotation \\
\hline S_PBL_C2971 & 936 & 3135.5 & 2.1 & 1466.5 & ABB97549.1 Sporamin A precursor [lpomoea batatas] \\
\hline S_PBL_C9555 & 931 & 1843.7 & 2.1 & 862.3 & AAA33391.1 Sporamin A precursor \\
\hline S_PBL_C21625 & 450 & 672.6 & 1.1 & 629.1 & BAA00828.1 Beta-amylase [Ipomoea batatas] \\
\hline S_PBL_C2075 & 958 & 502.3 & 1.1 & 469.9 & not annotated \\
\hline S_PBL_C1053 & 954 & 455.5 & $1^{*}$ & 455.6 & AAA33390.1 Sporamin B \\
\hline S_PBL_C54 & 918 & 2525.6 & 6.4 & 393.8 & P10917.1 Sporamin A; Flags: Precursor \\
\hline S_PBL_C17697 & 844 & 753.0 & 2.1 & 352.2 & AAB52547.1 Sporamin precursor \\
\hline S_PBL_C18468 & 263 & 311.5 & $1^{*}$ & 311.5 & not annotated \\
\hline S_PBL_C15531 & 438 & 2823.1 & 11.8 & 240.1 & AAA33391.1 Sporamin A precursor \\
\hline S_PBL_C8154 & 557 & 238.5 & $1^{*}$ & 238.5 & not annotated \\
\hline S_PBL_C3494 & 967 & 252.6 & 1.1 & 236.3 & AAL55800.1 Sporamin [lpomoea batatas] \\
\hline S_PBL_C3628 & 759 & 195.5 & $1^{*}$ & 195.5 & ABB90968.1 Sporamin B precursor [lpomoea batatas] \\
\hline S_PBL_C12997 & 481 & 190.8 & $1^{*}$ & 190.8 & not annotated \\
\hline S_PBL_C2027 & 1665 & 186.1 & 1.1 & 174.1 & BAA02286.1 Beta-amylase [Ipomoea batatas] \\
\hline S_PBL_Irc44407 & 487 & 158.1 & $1^{*}$ & 158.1 & not annotated \\
\hline S_PBL_C25030 & 652 & 150.6 & $1^{*}$ & 150.6 & $\begin{array}{l}\text { XP_001677106.1 Hypothetical protein CBG16734 } \\
\text { [Caenorhabditis briggsae AF16] }\end{array}$ \\
\hline S_PBL_C12797 & 567 & 134.7 & $1^{*}$ & 134.7 & not annotated \\
\hline S_PBL_C21769 & 669 & 131.0 & $1^{*}$ & 131.0 & not annotated \\
\hline S_PBL_Irc53020 & 508 & 386.3 & 3.2 & 120.5 & not annotated \\
\hline S_PBL_Irc25060 & 898 & 128.2 & 1.1 & 119.9 & CAO23244.1 unnamed protein product [Vitis vinifera] \\
\hline S_PBL_C34561 & 465 & 117.9 & $1^{*}$ & 117.9 & XP_002298721.1 predicted protein [Populus trichocarpa] \\
\hline S_PBL_C9857 & 630 & 581.8 & 5.3 & 108.9 & not annotated \\
\hline S_PBL_C3428 & 1350 & 577.2 & 5.3 & 108.0 & not annotated \\
\hline S_PBL_C18337 & 561 & 1035.5 & 9.6 & 107.6 & AAX27911.1 unknown [Schistosoma japonicum] \\
\hline S_PBL_C3870 & 696 & 104.8 & 1.1 & 98.0 & ABB83474.1 Beta-expansin precursor [Solanum lycopersicum] \\
\hline S_PBL_C45358 & 560 & 96.3 & $1^{*}$ & 96.3 & CAO47717.1 unnamed protein product [Vitis vinifera] \\
\hline S_PBL_C4363 & 735 & 199.2 & 2.1 & 93.2 & not annotated \\
\hline S_PBL_C20072 & 900 & 91.7 & $1^{*}$ & 91.7 & P10965.1 Sporamin B; Flags: Precursor \\
\hline S_PBL_C3830 & 725 & 291.8 & 3.2 & 91.0 & EEF50674.1 conserved hypothetical protein [Ricinus communis] \\
\hline S_PBL_C7863 & 397 & 90.7 & $1^{*}$ & 90.7 & not annotated \\
\hline S_PBL_IrC52376 & 494 & 189.9 & 2.1 & 88.8 & not annotated \\
\hline S_PBL_C2723 & 895 & 93.5 & 1.1 & 87.5 & CAO43166.1 unnamed protein product [Vitis vinifera] \\
\hline S_PBL_C1850 & 702 & 182.4 & 2.1 & 85.3 & not annotated \\
\hline S_PBL_C15391 & 502 & 89.8 & 1.1 & 84.0 & not annotated \\
\hline S_PBL_C26497 & 974 & 83.3 & $1^{*}$ & 83.3 & XP_002327563.1 predicted protein [Populus trichocarpa] \\
\hline S_PBL_C48117 & 388 & 262.9 & 3.2 & 82.0 & P10933.1 Ferredoxin-NADP reductase, leaf isozyme, chloroplastic \\
\hline S_PBL_C20358 & 683 & 85.1 & 1.1 & 79.6 & CAO22863.1 unnamed protein product [Vitis vinifera] \\
\hline S_PBL_C3576 & 454 & 169.3 & 2.1 & 79.2 & not annotated \\
\hline S_PBL_C8690 & 813 & 75.8 & $1^{*}$ & 75.8 & AAU93595.1 HVA22-like protein c, putative [Solanum demissum] \\
\hline S_PBL_C7526 & 860 & 79.5 & 1.1 & 74.4 & not annotated \\
\hline S_PBL_IrC53818 & 757 & 4080.3 & 58.8 & 69.4 & $\begin{array}{l}\text { BAF47746.1 ADP-glucose pyrophosphorylase beta subunit IbAGPb1A } \\
\text { [lpomoea batatas] }\end{array}$ \\
\hline S_PBL_IrC52525 & 509 & 73.0 & 1.1 & 68.3 & XP_002274272.1 PREDICTED: hypothetical protein [Vitis vinifera] \\
\hline S_PBL_Irc30491 & 523 & 145.9 & 2.1 & 68.3 & P27598.1 Starch phosphorylase L \\
\hline
\end{tabular}


Table 1 Top 70 up-regulated contigs in initiating storage roots compared to fibrous roots (Continued)

\begin{tabular}{|c|c|c|c|c|c|}
\hline S_PBL_C41294 & 520 & 68.3 & 1.1 & 63.9 & not annotated \\
\hline S_PBL_C32227 & 418 & 63.6 & $1^{*}$ & 63.6 & not annotated \\
\hline S_PBL_C54084 & 609 & 135.6 & 2.1 & 63.4 & not annotated \\
\hline S_PBL_C31475 & 869 & 66.4 & 1.1 & 62.1 & not annotated \\
\hline S_PBL_IrC52682 & 527 & 66.4 & 1.1 & 62.1 & XP_002306992.1 predicted protein [Populus trichocarpa] \\
\hline S_PBL_C10411 & 496 & 64.5 & 1.1 & 60.4 & BAA31699.1 PKn2 [lpomoea nil] \\
\hline S_PBL_Irc27049 & 954 & 255.4 & 4.3 & 59.7 & not annotated \\
\hline S_PBL_C14744 & 705 & 63.6 & 1.1 & 59.5 & EEF31509.1 Ubiquitin-protein ligase bre-1, putative [Ricinus communis] \\
\hline S_PBL_C18129 & 413 & 1393.8 & 23.5 & 59.3 & $\begin{array}{l}\text { BAF47744.2 ADP-glucose pyrophosphorylase alpha subunit IbAGPa1 } \\
\text { [lpomoea batatas] }\end{array}$ \\
\hline S_PBL_C7810 & 531 & 123.5 & 2.1 & 57.8 & not annotated \\
\hline S_PBL_C14132 & 307 & 57.1 & $1^{*}$ & 57.1 & not annotated \\
\hline S_PBL_C1234 & 886 & 2045.8 & 36.3 & 56.3 & $\begin{array}{l}\text { BAF47746.1 ADP-glucose pyrophosphorylase beta subunit IbAGPb1A } \\
\text { [lpomoea batatas] }\end{array}$ \\
\hline S_PBL_c695 & 596 & 721.2 & 12.8 & 56.2 & P27598.1 Starch phosphorylase L \\
\hline S_PBL_C30129 & 726 & 56.1 & $1^{*}$ & 56.1 & AAP41026.1 NTA15 protein [Nicotiana tabacum] \\
\hline S_PBL_C12308 & 666 & 115.1 & 2.1 & 53.8 & P93262.1 Phosphoglucomutase, cytoplasmic \\
\hline S_PBL_C28076 & 644 & 53.3 & $1^{*}$ & 53.3 & CAO21883.1 unnamed protein product [Vitis vinifera] \\
\hline S_PBL_C16839 & 282 & 55.2 & 1.1 & 51.6 & not annotated \\
\hline S_PBL_C47419 & 537 & 55.2 & 1.1 & 51.6 & $\begin{array}{l}\text { EEF50087.1 Serine-threonine protein kinase, plant-type, putative } \\
\text { [Ricinus communis] }\end{array}$ \\
\hline S_PBL_C51126 & 492 & 661.3 & 12.8 & 51.6 & $\begin{array}{l}\text { BAF47744.2 ADP-glucose pyrophosphorylase alpha subunit IbAGPa1 } \\
\text { [lpomoea batatas] }\end{array}$ \\
\hline S_PBL_C19033 & 878 & 51.4 & $1^{*}$ & 51.4 & not annotated \\
\hline S_PBL_Irc28526 & 555 & 51.4 & $1^{*}$ & 51.4 & not annotated \\
\hline S_PBL_C7145 & 689 & 161.8 & 3.2 & 50.5 & not annotated \\
\hline S_PBL_C12588 & 493 & 49.6 & $1^{*}$ & 49.6 & not annotated \\
\hline S_PBL_IrC53425 & 456 & 211.4 & 4.3 & 49.4 & $\begin{array}{l}\text { BAF47746.1 ADP-glucose pyrophosphorylase beta subunit IbAGPb1A } \\
\text { [lpomoea batatas] }\end{array}$ \\
\hline S_PBL_C21020 & 403 & 103.8 & 2.1 & 48.6 & not annotated \\
\hline S_PBL_C1397 & 989 & 408.8 & 8.6 & 47.8 & $\begin{array}{l}\text { BAF47744.2 ADP-glucose pyrophosphorylase alpha subunit IbAGPa1 } \\
\text { [Ipomoea batatas] }\end{array}$ \\
\hline S_PBL_C19766 & 429 & 984.1 & 21.4 & 46.0 & P27598.1 Starch phosphorylase L \\
\hline
\end{tabular}

Expression values are expressed as normalized read number. 'Fold change' is calculated as ISR reads/FR reads. One annotation is given for each contig. Annotations of the two best hits for each contig (E value $\leq 1.0 \mathrm{E}-3)$ are available in Additional file 1. ISR, Initiating storage root, FR fibrous root. * Read counts were changed from zero to one to enable 'Fold change' calculation (avoid dividing by zero).

expression of genes involved in starch biosynthesis detected by our Illumina sequencing (Table 1). To the best of our knowledge, the accumulated results represent novel data with respect to early elevation in expression of starch biosynthesis genes as well as starch accumulation, marking the initial phase of SR development.

Regulators of meristematic tissue identity and maintenance, as well as of cell division, are up-regulated in ISRs

A contig representing a class I knotted1-like protein (S_PBL_c10411) homologous to Ipomoea nil PKn2 (knotted-like gene) mRNA (AB016000.1) and Ibkn4 of
Ipomoea batatas (AB016000.1; class-I knotted1-like) showed 60-fold higher expression in ISRs compared to FRs (Table 1). Knotted1-like homeobox (KNOX) transcription factors are regulators involved in the establishment and maintenance of plant meristems, such as the shoot apical meristem [[33] and references therein]. The cambium is a stem-like tissue [34]; the term cambium refers to one or several layers of initials, analogous to the stem cells proposed for other meristems [[35] and references therein]. Divisions of these initials then produce phloem or xylem mother cells, which in turn can undergo several rounds of cell division before differentiating [35]. Schrader 


\section{Starch levels in developing sweetpotato adventitious roots}

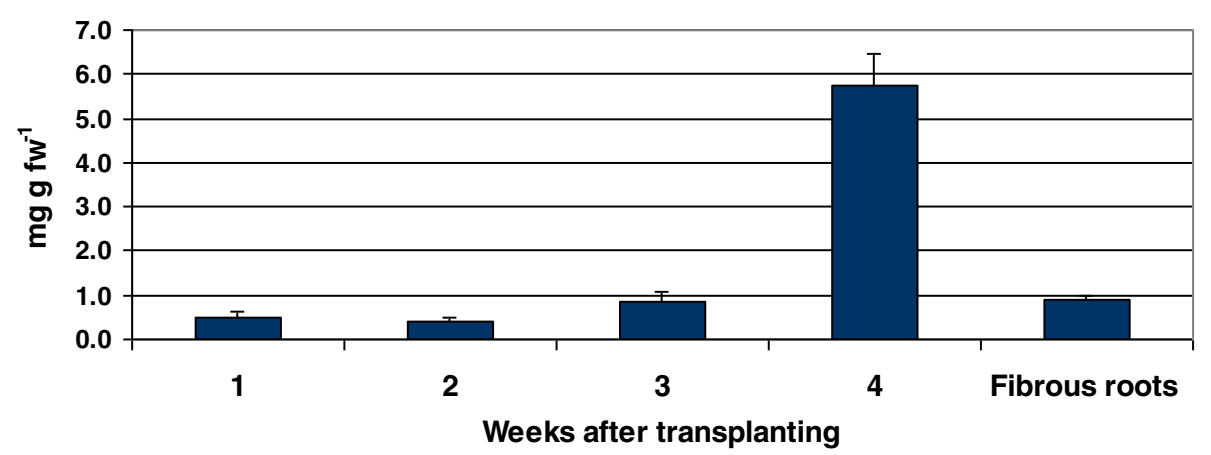

Figure 7 Starch levels in developing adventitious roots around the timing of storage root initiation. Root samples of Georgia Jet were pooled from five to seven plants at 1, 2, 3 and 4 weeks after transplanting, spanning the period of storage root (SR) initiation. Data represent an average of three biological replicates \pm SE. "Fibrous roots" represent fibrous root samples derived from plants at 8 weeks after transplanting (at least 4 weeks past the period of SR initiation).

et al. [35] identified molecular markers that characterize the cambial zone in poplar, including genes that regulate meristem identity and mark the cambium initials, and genes that control cell division and mark xylem mother cells. Of these genes, KNOX genes such as the poplar PttKNOX1, PttKNOX2 and PttKNOX6 showed high expression in cambial samples [35].

As indicated previously by Wilson and Lowe [5], and as demonstrated by us [7] for both the leading Israeli sweetpotato variety Georgia Jet and an important USA variety, Beauregard, initiation of SR formation is marked by the development of $\mathrm{AC}$ cells adjacent to xylem elements, starting 3 to 4 weeks after transplanting. Repeated division of these cambium cells leads to the formation of rows of thin-walled parenchyma cells that will form the storage tissue of the SR [7]. Several contigs homologous to KNOX genes were detected by us in the sweetpotato root transcriptome, with specific members exhibiting higher expression in ISRs compared to FRs (Table 2). It is interesting to note that all identified members of the class I knotted1-like proteins exhibited at least twofold higher expression in ISRs compared to FRs, while genes belonging to the class II knotted1-like family exhibited a versatile expression pattern (Table 2). KNOXI genes have been previously shown to be involved in the development of sweetpotato SRs [17,36], regulating cytokinin levels in that organ. Tanaka et al. [17] identified three different KNOXI gene fragments-ibkn1, ibkn2 and $i b k n 3$-in sweetpotato SR. Phylogenetic analysis of putative amino acid sequences showed that $i b k n 1$ is homologous to the SHOOT MERISTEMLESS (STM) gene of Arabidopsis thaliana, while $i b k n 2$ and $i b k n 3$ are homologous to the BP gene. Expressions of $i b k n 1, i b k n 2$ and $i b k n 3$ were faint or undetectable in fibrous, non-storage roots [17]. Mele eta al. [37] suggested in Arabidopsis that the BP gene regulates the lignin pathway, thus repressing premature cell differentiation. The class I knotted1-like genes found by us to be up-regulated in ISRs included $i b k n 2$ and $i b k n 3$ homologues as well as additional family members (Table 2).

Almost all of the cell-division-regulating genes that were detected in this work showed higher expression in ISRs compared to FRs, including genes encoding cyclin A-like and cyclin D-like proteins and five cyclin-dependent kinases (Table 2 and Additional file 5). These results are in agreement with the observed increase in the number of AC cells in sweetpotato ISR tissue sections (Figure 1) and with reports of accelerated cell division upon SR initiation in other sweetpotato varieties [36]. These results are novel and additional work is needed to characterize the spatial expression of these genes in root sections at different time points during and following SR initiation.

\section{Genes down-regulated during initiation of SR formation}

To identify root functions and processes that are downregulated during the development of FRs into SRs, we looked into genes represented by contigs that exhibit significantly higher fold-change expression in FRs compared to ISRs. The list of 70 contigs exhibiting highest differential expression in FRs compared to ISRs is summarized in Table 3. This list contains a relatively large number of non-annotated contigs, in addition to contigs that represent genes involved in root development and function as well as defense, such as a metallothioneinlike protein which has been shown in rice to be involved in root formation [38] and was found to exhibit a high read number (Table 3). Metallothioneins are cysteine-rich metal-binding proteins of low molecular mass that are mainly involved in maintaining metal homeostasis, metal detoxification and stress/defense responses [38]. Additional genes known to be involved in stress response 
Table 2 Expression, in initiating storage roots and fibrous roots, of genes involved in meristem- and cell-division regulation

\begin{tabular}{|c|c|c|c|c|}
\hline Contig & Length & ISR reads & FR reads & Annotation \\
\hline \multicolumn{5}{|c|}{ Meristem regulation } \\
\hline S_PBL_10411 & 496.0 & 64.5 & 1.1 & BAA31699.1 PKn2 [lpomoea nil] \\
\hline S_PBL_C8137 & 854.0 & 29.0 & 2.1 & BAF93479.1 Class-I knotted1-like homeobox protein IBKN2 [lpomoea batatas] \\
\hline S_PBL_C9098 & 625.0 & 52.4 & 25.7 & AAO33774.1 Knotted protein TKN4 [Lycopersicon esculentum] Class I knox \\
\hline S_PBL_C11762 & 744.0 & 171.2 & 16.0 & BAF93480.1 Class-I knotted1-like homeobox protein IBKN3 [lpomoea batatas] \\
\hline S_PBL_C31412 & 461.0 & 97.3 & 11.8 & BAF93480.1 Class-I knotted1-like homeobox protein IBKN3 [lpomoea batatas] \\
\hline S_PBL_IrC34271 & 455.0 & 2.8 & 44.0 & BO33481.1 Class II KNOX homeobox transcription factor [Medicago truncatula] \\
\hline S_PBL_C5315 & 807.0 & 30.9 & 25.7 & BAF95776.1 Class 2 knotted1-like protein [Nicotiana tabacum] \\
\hline S_PBL_c6714 & 747.0 & 6.5 & 3.2 & ABH03531.1 Class II knotted-like homeobox protein [Prunus persica] \\
\hline S_PBL_C12444 & 767.0 & 644.5 & 809.3 & NP_197904.1 KNAT3 [Arabidopsis thaliana] Class II KNOTTED1-like homeobox gene 3 \\
\hline \multicolumn{5}{|c|}{ Cell division regulation } \\
\hline S_PBL_C25867 & 444.0 & 18.7 & 0.0 & BAA20410.1 A-type cyclin [Catharanthus roseus] \\
\hline S_PBL_C32270 & 712.0 & 42.1 & 5.3 & AAV41032.1 Cyclin D-like protein [Nicotiana tabacum] \\
\hline S_PBL_C29324 & 408.0 & 213.3 & 336.7 & EEF32676.1 Cyclin-L2, putative [Ricinus communis] \\
\hline S_PBL_C3050 & 747.0 & 141.2 & 100.5 & AAR01224.1 Cyclin T1 [Medicago truncatula] \\
\hline S_PBL_C1272 & 1042.0 & 530.4 & 108.0 & BAE06268.1 Cyclin-dependent kinase A1 [Scutellaria baicalensis] \\
\hline S_PBL_C1655 & 489.0 & 44.9 & 3.2 & EEF38677.1 Cyclin-dependent kinases regulatory subunit, putative [Ricinus communis] \\
\hline S_PBL_C33143 & 467.0 & 35.5 & 6.4 & AAG01532.1 Cyclin-dependent kinase B1-1 [Nicotiana tabacum] \\
\hline S_PBL_C27090 & 768.0 & 112.3 & 54.5 & CAC51391.1 Cyclin dependent kinase C [Solanum lycopersicum] \\
\hline S_PBL_C28094 & 482.0 & 81.4 & 11.8 & ABN58480.1 Cyclin-dependent kinase [Actinidia chinensis] \\
\hline
\end{tabular}

Expression is given as the normalized number of reads derived from the lllumina sequencing data as detailed in 'Methods'. ISR, Initiating storage root, FR, Fibrous root.

(including to drought and salt stresses) and found to exhibit down-regulated expression in ISRs compared to FRs included an osmotin-like protein (a member of the class 5 pathogenesis-related proteins) and pathogenesis-related protein PR10a (Table 3; [39]), as well as a cysteine protease shown to be involved in senescence and the osmotic stress response [40].

In addition, contig S_PBL_c6142, which showed an over 100-fold higher read number in FRs compared with SRs, was found to exhibit significant homology to peroxidase 10-like mRNA (EC 1.11.1.7) of Vitis vinifera (3E-81) and Ricinus communis (2E-79), involved in the phenylpropanoid and lignin biosynthesis pathways. Angiosperm lignins are complex phenolic polymers that consist mostly of guaiacyl and syringyl units, together with small or trace amounts of $p$ hydroxyphenyl units. Monolignols are synthesized in the cytosol and transported to the cell wall, where their oxidation generates lignins [41]. From a functional point of view, lignins impart strength to cell walls, facilitate water transport, and impede the degradation of wall polysaccharides, thus acting as a major line of defense against pathogens, insects, and other herbivores [42]. In sweetpotato, Togari [4] proposed a direct link between lignification and SR initiation, suggesting that lignification inhibits SR development. The relationship between stele lignification and inability of adventitious roots to develop into SRs has also been observed by Wilson and Lowe [5], Belehu et al. [6] and Villordon et al. [7]. Togari [4] suggested that genetic and environmental factors influence the balance between cambium development and lignification, which in turn determines to a large degree the final SR yield. Indeed, looking into the expression levels of contigs representing additional lignin biosynthesis genes not included in Table 3, such as S_PBL_c158, S_PBL_c20480 and S_PBL_lrc53688, representing coumaroyl-CoA synthase, caffeoyl-CoA O-methyltransferase and cinnamyl alcohol dehydrogenase, respectively, more than sevenfold reduced expression in ISRs compared to FRs was detected (Additional files 5 and 6). This gene-expression pattern parallels the reduced lignification observed in tissue sections of ISRs compared to FRs (Figure 1; [7] and data not shown). In this context of potential cross talk between establishment and development of the cambium meristem on the one hand, and lignification on the other, it is interesting to note that data from studies on a specific peach class I knotted-like gene (knope1) suggest that KNOPE1 prevents cell lignification by repressing lignin genes during peach stem primary growth [43]. 
Table 3 Top 70 up-regulated contigs in fibrous roots compared to initiating storage roots

\begin{tabular}{|c|c|c|c|c|c|}
\hline Contig & Length & ISR reads & FR reads & Fold change & Annotation \\
\hline S_PBL_C4848 & 510 & 0.9 & 546.3 & 607.0 & not annotated \\
\hline S_PBL_C22976 & 665 & 2.8 & 756.9 & 270.3 & XP_002327317.1 Predicted protein [Populus trichocarpa] \\
\hline S_PBL_IrC52198 & 626 & 0.9 & 228.8 & 254.2 & AAK27968.1 Cysteine protease [lpomoea batatas] \\
\hline S_PBL_C1692 & 703 & $1^{*}$ & 251.2 & 251.2 & not annotated \\
\hline S_PBL_C14075 & 626 & $1^{*}$ & 232 & 232.0 & XP_001837463.1 Tubulin alpha chain [Coprinopsis cinerea okayama7\#130] \\
\hline S_PBL_C36764 & 703 & 3.7 & 856.3 & 231.4 & XP_002303479.1 predicted protein [Populus trichocarpa] \\
\hline S_PBL_C1452 & 539 & 0.9 & 206.3 & 229.2 & AAK27968.1 Cysteine protease [lpomoea batatas] \\
\hline S_PBL_C25084 & 735 & $1^{*}$ & 209.5 & 209.5 & CAO14793.1 unnamed protein product [Vitis vinifera] \\
\hline S_PBL_C2976 & 600 & 26.2 & 5316.4 & 202.9 & Q40157.1 Metallothionein-like protein type $2 \mathrm{~A}$ \\
\hline S_PBL_Irc52159 & 665 & 0.9 & 174.3 & 193.7 & not annotated \\
\hline S_PBL_C43111 & 446 & 0.9 & 168.9 & 187.7 & not annotated \\
\hline S_PBL_C39992 & 480 & $1^{*}$ & 187.1 & 187.1 & XP_001874004.1 predicted protein [Laccaria bicolor] \\
\hline S_PBL_C18994 & 719 & 20.6 & 3790.8 & 184.0 & not annotated \\
\hline S_PBL_c28403 & 834 & 0.9 & 164.6 & 182.9 & XP_001884841.1 Delta 9-fatty acid desaturase protein [Laccaria bicolor] \\
\hline S_PBL_C30965 & 742 & 0.9 & 153.9 & 171.0 & CAO23367.1 unnamed protein product [Vitis vinifera] \\
\hline S_PBL_C29652 & 772 & 0.9 & 151.8 & 168.7 & XP_002304020.1 predicted protein [Populus trichocarpa] \\
\hline S_PBL_C12455 & 525 & 34.6 & 5763.2 & 166.6 & Q40157.1 Metallothionein-like protein type $2 \mathrm{~A}$ \\
\hline S_PBL_C5087 & 783 & 2.8 & 465 & 166.1 & not annotated \\
\hline S_PBL_C18075 & 609 & 4.7 & 767.6 & 163.3 & not annotated \\
\hline S_PBL_C44512 & 388 & 0.9 & 145.4 & 161.6 & XP_002279608.1 hypothetical protein [Vitis vinifera] \\
\hline S_PBL_C19899 & 443 & 0.9 & 144.3 & 160.3 & not annotated \\
\hline S_PBL_C14724 & 532 & $1^{*}$ & 156.1 & 156.1 & CAN80314.1 hypothetical protein [Vitis vinifera] \\
\hline S_PBL_C30195 & 672 & 0.9 & 136.8 & 152.0 & not annotated \\
\hline S_PBL_c8847 & 687 & $1^{*}$ & 150.7 & 150.7 & XP_002330606.1 predicted protein [Populus trichocarpa] \\
\hline S_PBL_c6142 & 645 & 1.9 & 275.8 & 145.2 & XP_002263033 Peroxidase 10-like [Vitis vinifera] \\
\hline S_PBL_C11711 & 510 & 7.5 & 1082.9 & 144.4 & XP_001880208.1 Fumarate reductase [Laccaria bicolor] \\
\hline S_PBL_C35569 & 653 & 3.7 & 533.5 & 144.2 & not annotated \\
\hline S_PBL_C337 & 838 & 27.1 & 3771.6 & 139.2 & AAL79832.2 Osmotin-like protein [Solanum nigrum] \\
\hline S_PBL_C50215 & 567 & 1.9 & 261.9 & 137.8 & not annotated \\
\hline S_PBL_Irc32508 & 540 & 0.9 & 124 & 137.8 & not annotated \\
\hline S_PBL_C12800 & 554 & 1.9 & 260.8 & 137.3 & XP_001881080.1 Histone 2A [Laccaria bicolor] \\
\hline S_PBL_C13942 & 648 & 3.7 & 504.6 & 136.4 & XP_001888404.1 Sphingolipid C9-methyltransferase [Laccaria bicolor] \\
\hline S_PBL_C837 & 483 & 1.9 & 258.7 & 136.2 & not annotated \\
\hline S_PBL_C14357 & 689 & 3.7 & 488.6 & 132.1 & EAY93058.1 hypothetical protein OsI_14861 [Oryza sativa Indica Group] \\
\hline S_PBL_C44050 & 575 & 1.9 & 242.7 & 127.7 & $\begin{array}{l}\text { XP_001834214.1 hypothetical protein CC1G_09714 } \\
\text { [Coprinopsis cinerea okayama7\#130] }\end{array}$ \\
\hline S_PBL_C47284 & 674 & 0.9 & 114.4 & 127.1 & EEF39207.1 Rhicadhesin receptor precursor, putative [Ricinus communis] \\
\hline S_PBL_C29183 & 616 & 0.9 & 113.3 & 125.9 & not annotated \\
\hline S_PBL_C15554 & 601 & 13.1 & 1644.2 & 125.5 & Q40157.1 Metallothionein-like protein type $2 \mathrm{~A}$ \\
\hline S_PBL_C28805 & 440 & $1^{*}$ & 122.9 & 122.9 & CAO44491.1 unnamed protein product [Vitis vinifera] \\
\hline S_PBL_C9181 & 557 & 1.9 & 230.9 & 121.5 & AAY87888.1 Glutathione peroxidase [Taiwanofungus camphoratus] \\
\hline S_PBL_IrC53720 & 379 & 52.4 & 6313.8 & 120.5 & not annotated \\
\hline S_PBL_C18857 & 626 & $1^{*}$ & 117.6 & 117.6 & AAK27968.1 Cysteine protease [lpomoea batatas] \\
\hline S_PBL_C5350 & 526 & 1.9 & 221.3 & 116.5 & not annotated \\
\hline
\end{tabular}


Table 3 Top 70 up-regulated contigs in fibrous roots compared to initiating storage roots (Continued)

\begin{tabular}{|c|c|c|c|c|c|}
\hline S_PBL_C41001 & 434 & 0.9 & 103.7 & 115.2 & XP_572855.1 Polysaccharide synthase [Cryptococcus neoformans] \\
\hline S_PBL_C17858 & 525 & $1^{*}$ & 113.3 & 113.3 & not annotated \\
\hline S_PBL_C30000 & 488 & 0.9 & 101.6 & 112.9 & $\begin{array}{l}\text { XP_001838960.1 hypothetical protein CC1G_05513 } \\
\text { [Coprinopsis cinerea okayama7\#130] }\end{array}$ \\
\hline S_PBL_C25576 & 582 & 0.9 & 100.5 & 111.7 & not annotated \\
\hline S_PBL_c6293 & 718 & 12.2 & 1345.9 & 110.3 & not annotated \\
\hline S_PBL_C37132 & 470 & 0.9 & 95.1 & 105.7 & not annotated \\
\hline S_PBL_C3863 & 605 & 1.9 & 198.8 & 104.6 & CAN80314.1 hypothetical protein [Vitis vinifera] \\
\hline S_PBL_C12972 & 545 & 15.9 & 1651.7 & 103.9 & AAL16409.1 Pathogenesis-related protein PR10a [Nicotiana tabacum] \\
\hline S_PBL_C2399 & 892 & 2.8 & 290.8 & 103.9 & CAO22665.1 unnamed protein product [Vitis vinifera] \\
\hline S_PBL_C7735 & 565 & 1.9 & 196.7 & 103.5 & NP_200248.1 Late embryogenesis abundant protein-related [Arabidopsis thaliana] \\
\hline S_PBL_C28770 & 429 & 1.9 & 195.6 & 102.9 & not annotated \\
\hline S_PBL_C33041 & 444 & 1.9 & 194.6 & 102.4 & not annotated \\
\hline S_PBL_C27326 & 782 & 6.5 & 664.9 & 102.3 & XP_001890326.1 GPA1 heterotrimeric G-protein alpha subunit [Laccaria bicolor] \\
\hline S_PBL_C18989 & 540 & 1.9 & 193.5 & 101.8 & AAK27968.1 Cysteine protease [lpomoea batatas] \\
\hline S_PBL_c6361 & 637 & 8.4 & 855.2 & 101.8 & XP_001880208.1 Fumarate reductase [Laccaria bicolor] \\
\hline S_PBL_C15414 & 674 & $1^{*}$ & 100.5 & 100.5 & AAL14199.1 Cysteine proteinase precursor [lpomoea batatas] \\
\hline S_PBL_C15414 & 674 & $1^{*}$ & 100.5 & 100.5 & AAL14199.1 Cysteine proteinase precursor [lpomoea batatas] \\
\hline S_PBL_C18957 & 652 & 1.9 & 190.3 & 100.2 & not annotated \\
\hline S_PBL_C12342 & 570 & $1^{*}$ & 98.4 & 98.4 & not annotated \\
\hline S_PBL_C10766 & 501 & $1^{*}$ & 94.1 & 94.1 & not annotated \\
\hline S_PBL_C40440 & 403 & $1^{*}$ & 94.1 & 94.1 & not annotated \\
\hline S_PBL_C11884 & 622 & $1^{*}$ & 93 & 93 & XP_002273655.1 PREDICTED: hypothetical protein [Vitis vinifera] \\
\hline S_PBL_IrC28732 & 494 & $1^{*}$ & 87.7 & 87.7 & not annotated \\
\hline S_PBL_C24730 & 463 & $1^{*}$ & 86.6 & 86.6 & not annotated \\
\hline S_PBL_C32483 & 699 & $1^{*}$ & 86.6 & 86.6 & not annotated \\
\hline S_PBL_C14615 & 660 & $1^{*}$ & 84.5 & 84.5 & EEF31626.1 Kiwellin, putative [Ricinus communis] \\
\hline S_PBL_C28823 & 729 & $1^{*}$ & 83.4 & 83.4 & $\begin{array}{l}\text { XP_001831241.1 hypothetical protein CC1G_00788 } \\
\text { [Coprinopsis cinerea okayama7\#130] }\end{array}$ \\
\hline
\end{tabular}

Expression values are expressed as normalized read number. 'Fold change' is calculated as FR reads/ISR reads. One annotation is given for each contig. Annotations of the two best hits for each contig (E value $\leq 1.0 \mathrm{E}-3$ ) are available in Additional file 1. ISR, Initiating storage root, FR, Fibrous root. * - Read counts were changed from zero to one to enable 'Fold change' calculation.

\section{Validation of differential expression between ISRs and FRs revealed by the use of Illumina-based sequencing at the cDNA level}

The validity of the expression differences detected by Illumina-based sequencing was examined at the RNA level, using on-line quantitative (q) RT-PCR. Results for nine selected contigs are presented (Figure 8), representing five groups of genes that were found to be differentially expressed by the Illumina results: a gene encoding sporamin, a sweetpotato storage protein accounting for about $60 \%$ of total soluble proteins, genes encoding enzymes involved in starch biosynthesis (AGPase and granule-bound starch synthase 1-GBBS1), genes regulating meristem identity (homologous to class-I knotted-like homeobox proteins Ibkn2 and Ibkn3), a gene involved in cell division regulation (cyclin-dependent kinase-CDK) and genes encoding enzymes of lignin biosynthesis (4 coumaroyl-CoA synthase 1-4CL; caffeoyl-CoA O-methyltransferase 2-CCoAOMT; cinnamyl alcohol dehydrogenase-CAD). Results are presented as relative expression values of the respective genes in the ISR sample relative to their expression in the FR sample. In all cases tested, the qRT-PCR analyses confirmed the results of the Illumina analyses (Figure 8), namely elevated expression of gene sequences involved in starch biosynthesis and sporamin accumulation, of the gene encoding CDK and of genes belonging to the class-I knotted-like homeobox protein family, and down-regulation of genes encoding enzymes of lignin biosynthesis in ISRs. However, some of the relative expression values differed between the 


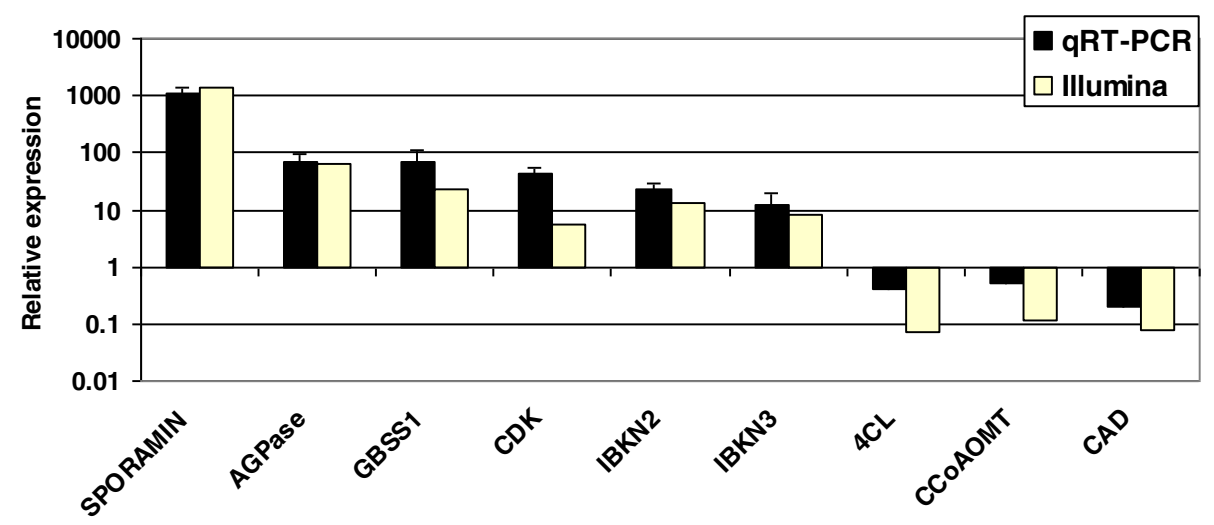

Figure 8 Validation by quantitative on-line RT-PCR analyses of the differential expression between sweetpotato initiating storage and fibrous roots revealed by the use of Illumina-based sequencing at the cDNA level. Expression levels were measured in initiating storage root (ISR) and fibrous root (FR) samples of Georgia Jet sweetpotato variety, using at least four biological replicates. Each replicate consisted of CDNA representing pooled root tissue from 30 plants. Quantitative RT-PCR was performed and values were normalized relative to the expression levels of 18S rRNA in the same cDNA sample. Expression data are the means ( \pm SE) of at least four replicates and are presented as relative expression values of the respective gene in the ISR sample relative to its expression in the FR sample (ISR/FR). The $Y$ axis has a logarithmic scale. ADP glucose pyrophosphorylase - AGPase; coumaroyl-CoA synthase - 4CL; caffeoyl-CoA O-methyltransferase - CCoAOMT; cinnamyl alcohol dehydrogenase - CAD.

two expression analysis methods. For example, relative expression values detected by the Illumina and qRT-PCR methods for CDK, 4CL and CAD were 5.6, 0.07, 0.08 and $44.8,0.4$ and 0.2 , respectively. It should be noted that the Illumina-based expression results were derived from one biological replicate of the ISR sample and one biological replicate of the FR sample (each sample consisting of cDNA representing pooled root tissue of 30 plants), while the qRT-PCR results were derived from at least four biological replicates (each sample consisting of cDNA representing pooled root tissue of 30 plants). Overall, the qRT-PCR results validated the Illumina-based cDNA sequencing results, allowing for the interpretation of global gene expression trends. Independent validations are still required to accurately measure the expression of each gene of interest.

\section{Analysis of over-represented GO terms in the subset of differentially expressed contigs, in either ISRs or FRs, relative to the root transcriptome database}

The differentially expressed contigs in ISRs and FRs were analyzed for GO-category enrichment relative to the root transcriptome database using AgriGO [44]. The results for the up-regulated contigs in ISRs are presented in Figure 9 and Additional files 7 and 8. The up-regulated contigs in ISRs were found to contain 21 significantly enriched $(\mathrm{FDR} \leq 0.05) \mathrm{GO}$ functional terms in the biology process category, including three 'level 3' and four 'level 4' terms ("carbohydrate metabolic process", GO:0005975; "alcohol metabolic process", GO:0006066; "lipid localization", GO:0010876 as well as "photosynthesis", GO:0015979; "lipid transport", GO:0006869; "cellular carbohydrate metabolic process", GO:0044262; "nodulation”, GO:0009877, respectively). The highly enriched $(\mathrm{FDR}=0.0003)$ "carbohydrate metabolic process" term included several contigs of starch phosphorylase, phosphoglucomutase, starch branching enzyme and 1,3- $\beta$-glucosidase, and its daughter terms included "monosaccharide metabolic process" (GO:0005996) and "starch biosynthetic process" (GO: 0019252; including several contigs of ADP glucose pyrophosphorylase and starch synthase). The results thus point to enrichment in ISRs of carbohydrate metabolism and starch biosynthesis functions, in accordance with the biochemical analysis results indicating starch accumulation at the time of SR initiation (Figure 7). An additional and interesting enriched term was "protein folding" (GO:0006457), a 'level 7' term that included contigs of cyclophilin, peptidyl-prolyl cis/trans isomerase, calnexin CONSTANS interacting protein 3 , heat-shock protein and chaperones, and was connected to the term "protein thiol-disulfide exchange" (GO:0006467).

As for molecular function, the up-regulated contigs belonged to 13 significantly enriched GO terms, including the 'level 1' term "nutrient reservoir activity" (GO:0045735), containing several contigs of sporamin A and sporamin B precursors, the 'level 2' terms "lipid binding" (GO:0008289), "enzyme inhibitor activity" (GO:0004857), and the 'level 3' terms "transferase activity, transferring sulfur-containing groups" (GO:0016782) and "peptidase inhibitor activity" (GO:0030414). The most significantly enriched term was the 'level 6' term "phosphofructokinase activity" (GO:0008443). "Glucose-1-phosphate adenylyltransferase (GO:0008878), containing several contigs of ADP-glucose pyrophosphorylase subunits $\alpha$ and $\beta$, was also significantly enriched 


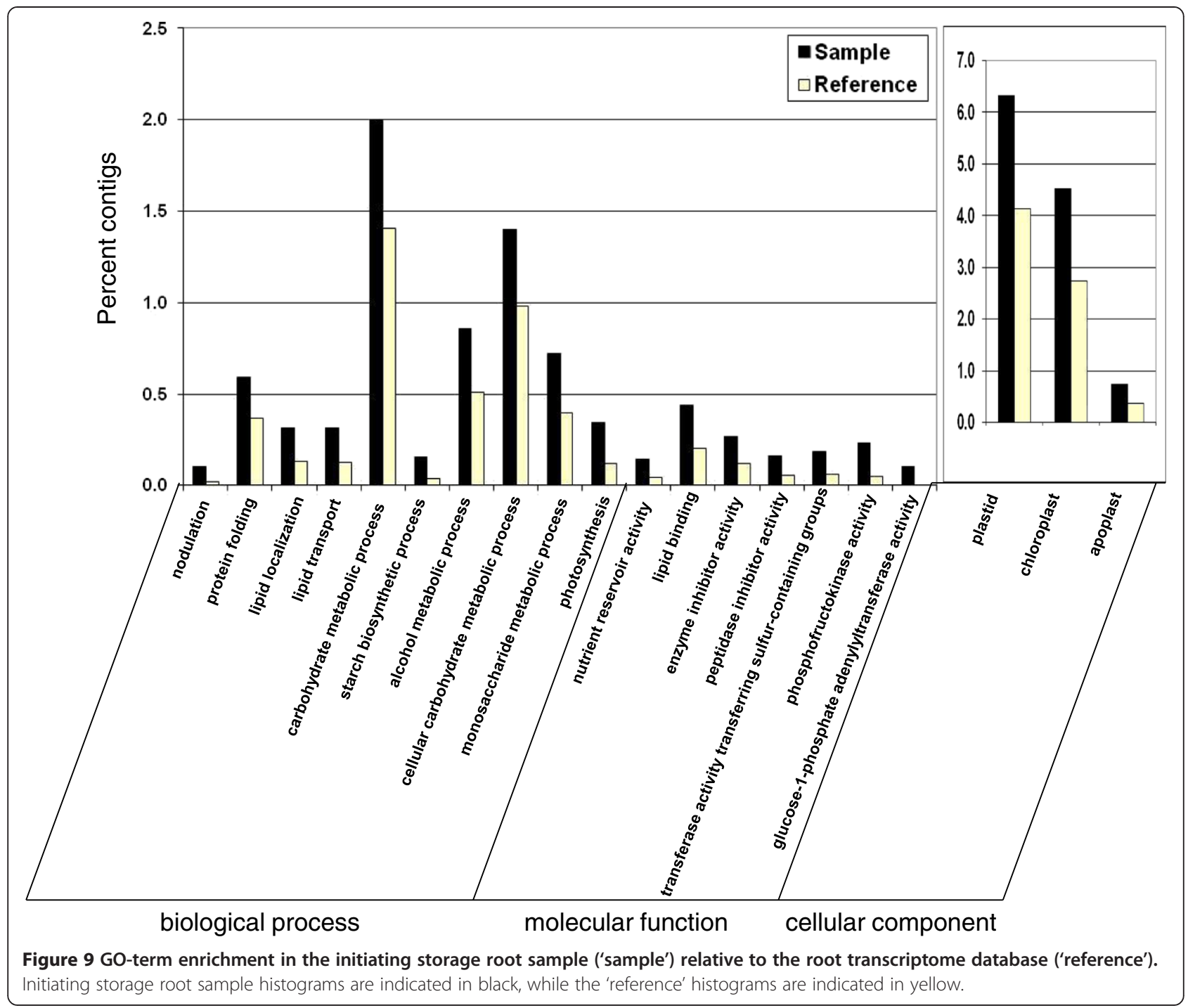

(FDR $=0.028)$. The GO terms of "plastid" (GO:0009536) and "chloroplast" (GO:0009507) and their daughter terms were the most highly enriched in the cellular component category (with FDR values of 4.03E-26 and 4.02E-25, respectively), in accordance with involvement of amyloplasts in SR starch accumulation. The "apoplast" term (GO:0048046) was also found to be significantly enriched in the ISR sample (Figure 9).

The up-regulated contigs in FRs were found to represent a larger number of different enriched GO functional categories $(\mathrm{FDR} \leq 0.05)$ compared to ISRs (Figure 10 and Additional files 9 and 10). Among the enriched terms represented by the up-regulated contigs in FRs, a total of 74 terms were included in the BP category, containing the 'level 2' terms "oxidation reduction" (GO:0055114), "small molecule metabolic process" (GO:0044281), "response to stress" (GO:0006950), "response to biotic stimulus" (GO:0009607), "response to chemical stimulus" (GO:0042221) and "secondary metabolic process" (GO:0019748), and the 'level 3' terms "response to endogenous stimulus" (GO: GO:0009719) and "transport" (GO:0006810), which in turn included "transmembrane transport" (GO:0055085). The enriched "secondary metabolic process" term exhibited high enrichment in "phenylpropanoid metabolic process" (GO:0009698; containing several contigs of coumaroyl CoA synthase and phenylalanine ammonia lyase), "phenylpropanoid biosynthetic process" (GO:0009699), "coumarin metabolic process" (GO:0009804), "coumarin biosynthetic process" (GO:0009805; containing contigs of p-coumaroyl quinate/shikimate 3'-hydroxylase, caffeoyl coenzyme A 3o-methtyl transferase), "lignin metabolic process" (GO: 0009808), "phenylpropanoid catabolic process" (GO: 0046271 see Figure 10) and "lignin catabolic process" (GO:0046274). An interesting group of enriched functional terms (FDR $\leq 0.032$ ) included: "meristem determinacy" 


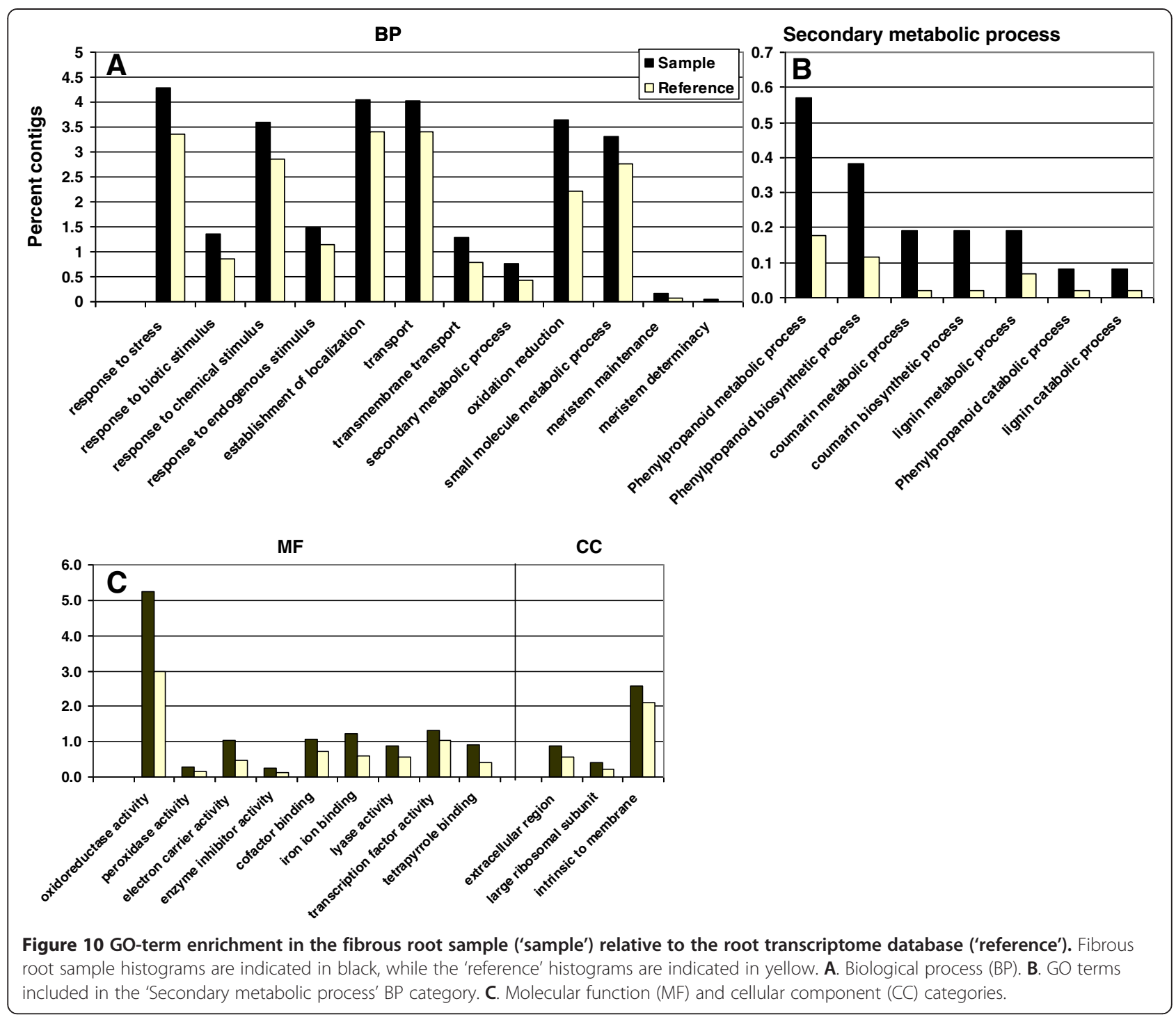

(GO:0010022), "meristem maintenance" (GO:0010073) and "inflorescence meristem growth" (GO:0010450), which contained several contigs representing at least three ultrapetala 1-like proteins. Ultrapetala 1-like protein is a putative transcription factor that acts as a key negative regulator of cell accumulation in Arabidopsis shoot and floral meristems [45]. The higher-plant shoot apical meristem is a dynamic structure that continuously produces cells which become incorporated into new leaves, stems and flowers. The maintenance of a constant flow of cells through the meristem depends on coordination of two antagonistic processes: self-renewal of the stem cell population and initiation of the lateral organs. This coordination is stringently controlled by gene networks that contain both positive and negative components. Carles et al. $[45,46]$ defined the ULTRAPETALA1 (ULT1) gene as a key negative regulator of cell accumulation in Arabidopsis shoot and floral meristems, because mutations in ULT1 caused enlargement of inflorescence and floral meristems, the production of supernumerary flowers and floral organs, and a delay in floral meristem termination.

In the MF category, the up-regulated contigs belonged to 78 significantly enriched GO terms, including electron carrier, transporter, antioxidant and catalytic activities, and binding. Catalytic activity included "oxidoreductase activity" (GO:0016491), which in turn included "oxidoreductase activity, acting on diphenols and related substances as donors, oxygen as acceptor" (GO:0016682), "oxidoreductase activity, acting on paired donors, with incorporation or reduction of molecular oxygen, NADH or NADPH as one donor, and incorporation of one atom of oxygen" (GO:0016709) and "trans-cinnamate 4 monooxygenase activity" (GO:0016710), as well as "oxidoreductase activity, acting on paired donors, with incorporation or reduction of molecular oxygen, 2-oxoglutarate as one donor, and incorporation of one atom each of oxygen into both 
donors" (GO:0016706), "naringenin 3-dioxygenase activity" (GO:0045486) and "leucocyanidin oxygenase activity" (GO:0050589). Catalytic activity also included transferase activity which, in turn, included "glucosyltransferase activity" (GO:0046527), "anthocyanidin 3-O-glucosyltransferase activity" (GO:0047213) and "transferase activity, transferring acyl groups other than amino-acyl groups" (GO:0016747) and "hydroxycinnamoyltransferase activity" (GO:0050734). Binding included "heme binding" (GO:0020037), "cofactor binding" (GO:0048037), including "FAD binding" (GO:0050660) as well as "transcription factor activity" (GO:0003700).

It is interesting to note that the enriched term "transcription factor activity" included several ethylene-responsive factors, such as a homologue of tomato ethylene responsive transcriptional coactivator (MBF1), AP2/ERF domaincontaining contigs, homologues of dehydration-responsive element binding protein, and ethylene-responsive transcription factor 2 (ERF2) and ERF5, indicating involvement of ethylene in sweetpotato root development. The growth of secondary xylem and phloem depends on the division of cells in the vascular cambium and results in an increase in the diameter of the root. Results of genome-wide expression profiling of xylem and phloem cambium isolated from the root hypocotyl of Arabidopsis suggested a role for several members of the AP2 as well as MYB transcription factor families, in addition to other transcription factors, as regulators of xylem or phloem cell differentiation and activity [47]. This "transcription factor activity" GO term also contained R2R3 MYB class transcription factor homologues, several GRAS2 and GRAS10 homologues, two BEL1 homeotic protein homologues and a CONSTANS-like protein homologue. Members of the GRAS family, such as the short-root protein, are key regulators of root radial patterning [[20] and references therein], meristem maintenance and asymmetric cell division [[20] and references therein], and have been found to be differentially expressed in SRs of the sweetpotato cv. Xushu [20].

The GO terms "large ribosomal subunit" (GO:0015934), "extracellular region" (GO:0005576) and "intrinsic to membrane" (GO:0031224) were found to be significantly enriched in the CC category (Figure 10C).

\section{Analysis of KEGG pathway enrichment in differentially expressed contigs of either ISRs or FRs, relative to the root transcriptome database}

The differentially expressed contigs in ISRs and FRs were analyzed for KEGG pathway enrichment, relative to the rest of the root transcriptome database, using Fisher's Exact Test and FDR correction [48], and the results are presented in Table 4. Four and fifteen pathways were found to be significantly enriched $(F D R \leq 0.05)$ in ISR and FR, respectively.
Looking into KEGG-enriched pathways in ISRs, enrichment of sulfur metabolism was apparent, with 10 enzymes exhibiting up-regulated expression in ISRs compared to FRs (Additional file 11), including contigs representing sulfite reductase (ferredoxin). Interestingly, sulfite reductase has been recently suggested as a candidate gene with potential function in cassava SR formation [49]. Reduced levels of sulfite reductase in the Arabidopsis mutant sirl with decreased activity of the enzyme led to decreased hexose and starch contents [50]. It is thus tempting to suggest that sulfite reductase may also have a role in the control of SR starch accumulation during SR initiation. The glycolysis/gluconeogenesis pathway was also found to be significantly enriched in ISRs (FDR $=0.051$; Table 4). In cassava, regulatory changes in the glycolysis/gluconeogenesis pathway were demonstrated following SR development [51]. Down-regulation of enolase, L-lactate dehydrogenase and aldehyde dehydrogenase was suggested in cassava to slow down the entry of carbon into the citrate cycle, pyruvate metabolism and propanoate metabolism, leading to less glucose-6P converted to glycerate-3P and glucose-1P, and in most of the glucose-6P being transported into the amyloplast for starch and sucrose synthesis [51]. In sweetpotato, over twofold down-regulation of several contigs of pyruvate decarboxylase and lactate dehydrogenase was detected following initiation of SR formation (Additional file 5), as well as more than twofold up-regulation of contigs of phosphoglucomutase (catalyzing the reversible interconversion between glucose-6P and glucose-1P, the latter serving as a substrate for ADP-Glc pyrophosphorylase, the first committed step in the starch biosynthesis pathway), indicating the possibility of similar regulation.

Among the significantly enriched pathways in FRs, high enrichment was observed for the phenylpropanoid biosynthesis pathway (Table 4). The first three biosynthetic reactions in this pathway, referred to as the general phenylpropanoid pathway, produce p-coumaroyl CoA, which is a major branch-point metabolite between the production of the flavonoids and the pathway that produces monolignols, lignans and hydroxy-cinnamate conjugates [[52] and references therein]. The first of these reactions is the deamination of phenylalanine by phenylalanine ammonia-lyase (PAL) to generate trans-cinnamic acid. Cinnamic acid is then para-hydroxylated by cinnamate 4-hydroxylase $(\mathrm{C} 4 \mathrm{H})$ to produce $\mathrm{p}$-coumaric acid [[52] and references therein], which is then activated to its corresponding CoA thioester by 4-coumarate CoA ligase (4 coumaroyl-CoA synthase; 4CL). All phenylalaninederived units destined to be incorporated into the lignin polymer must be hydroxylated by $\mathrm{C} 4 \mathrm{H}$, because the $\mathrm{p}$ hydroxy group is required for the activation of monolignols to their corresponding free radicals, and for polymerization into lignin. The phenylpropanoid biosynthesis pathway map 
Table 4 KEGG pathway enrichment in initiating storage roots and fibrous roots, relative to the rest of the root transcriptome database

\begin{tabular}{|c|c|c|c|}
\hline Sample & Pathway term & FDR & KEGG map \\
\hline \multirow[t]{15}{*}{ Fibrous roots } & Flavonoid biosynthesis & $2.3 \mathrm{E}-17$ & map00941 \\
\hline & Phenylpropanoid biosynthesis & $5.3 \mathrm{E}-15$ & map00940 \\
\hline & Anthocyanin biosynthesis & $1.2 \mathrm{E}-11$ & map00942 \\
\hline & Stilbenoid, diarylheptanoid and gingerol biosynthesis & $3.8 \mathrm{E}-10$ & map00945 \\
\hline & Drug metabolism - other enzymes & $1.1 \mathrm{E}-05$ & map00983 \\
\hline & Isoquinoline alkaloid biosynthesis & $1.7 \mathrm{E}-04$ & map00950 \\
\hline & Linoleic acid metabolism & $6.7 \mathrm{E}-04$ & map00591 \\
\hline & Phenylalanine metabolism & $5.3 \mathrm{E}-03$ & map00360 \\
\hline & alpha-Linolenic acid metabolism & 7.0E-03 & map00592 \\
\hline & Zeatin biosynthesis & $1.1 \mathrm{E}-02$ & map00908 \\
\hline & Cysteine and methionine metabolism & $1.2 \mathrm{E}-02$ & map00270 \\
\hline & Flavone and flavonol biosynthesis & $2.4 \mathrm{E}-02$ & map00944 \\
\hline & Propanoate metabolism & $2.5 \mathrm{E}-02$ & map00640 \\
\hline & Ubiquinone and other terpenoid quinone biosynthesis & $4.4 \mathrm{E}-02$ & map00130 \\
\hline & Betalain biosynthesis & $5.2 \mathrm{E}-02$ & map00965 \\
\hline \multirow[t]{4}{*}{ Initiating storage roots } & Galactose metabolism & 0.000 & map00052 \\
\hline & Sulfur metabolism & 0.001 & map00920 \\
\hline & Fructose and mannose metabolism & 0.001 & map00051 \\
\hline & Glycolysis/Gluconeogenesis & 0.051 & map00010 \\
\hline
\end{tabular}

Enrichment analysis was done using Fisher's Exact Tests. P-value is given as an adjusted value following FDR correction (FDR $\leq 0.05)$.

is presented in Figure 11, with the enzymes exhibiting up-regulated expression in ISRs and FRs marked in green and red, respectively. Over twofold up-regulation in FRs of contigs representing $\mathrm{C} 4 \mathrm{H}$ and $4 \mathrm{CL}$, as well as of contigs of coniferyl-alcohol glucosyltransferase was apparent (Figure 11 and Table 5). In addition, high expression of PAL was detected in the FR sample, whereas an over fourfold reduction in read number was observed in the ISR sample (Additional file 5). The presence of several contigs representing these enzymes may indicate the presence of isoenzymes. 4CL catalyzes the formation of CoA esters of caffeic acid, ferulic acid, 5-hydroxyferulic acid, and sinapic acid, in addition to p-coumaric acid [[53] and references therein]. The plethora of additional potential substrates may explain why there are many $4 \mathrm{CL}$ isoenzymes in most plants. In addition to the different substrate specificities, the genes may have a distinct spatiotemporal expression pattern [[53] and references therein]. Looking into the read number of contigs representing genes of the lignin pathway, such as cinnamyl alcohol dehydrogenase (CAD), more than fivefold lower expression was detected in the ISR vs. FR sample (Table 5 and Additional file 5). Taken together, the results indicate down-regulation in the expression of key genes of the phenylpropanoid biosynthesis pathway upon the change in root fate from FR to a storage organ, which may be responsible for the significant reduction in lignin levels
(Figure 1), representing novel data not previously described in sweetpotato. Indeed, it has been demonstrated in Arabidopsis and tobacco that down-regulating 4CL results in reduced lignin content [54-56]. Hu et al. [57] showed that down-regulating the expression of $4 \mathrm{CL}$ in transgenic aspen (Populus tremuloides Michx.) by antisense inhibition causes up to $45 \%$ reduction in lignin. Reductions in lignin content in Arabidopsis plants carrying a mutation in the second enzyme of this pathway, $\mathrm{C} 4 \mathrm{H}$, were shown to accumulate decreased levels of several different classes of phenylpropanoid end products and to exhibit reduced lignin deposition, altered lignin monomer content and a collapsed xylem phenotype [52].

Cross-talk between primary and secondary metabolism is well documented [58]. To activate the lignin biosynthesis pathway, carbon flow should be delivered from carbohydrate metabolism into the phenylpropanoid pathway by producing sufficient phenylalanine via the shikimate pathway. 3-Deoxy-D-arabino-heptulosonate-7-phosphate synthase (EC 2.5.1.54) catalyzes the first step of the shikimate pathway, using phosphoenolpyruvate and erythrose 4phosphate derived from glycolysis and the pentose phosphate pathway, respectively. It was thus interesting to note that more than fourfold down-regulation in read number of six contigs (S_PBL_c17757, S_PBL_lrc54453, S_PBL_lrc54372, S_PBL_lrc55028, S_PBL_c7135, S_PBL_ c8050) representing this gene, was detected in ISR 


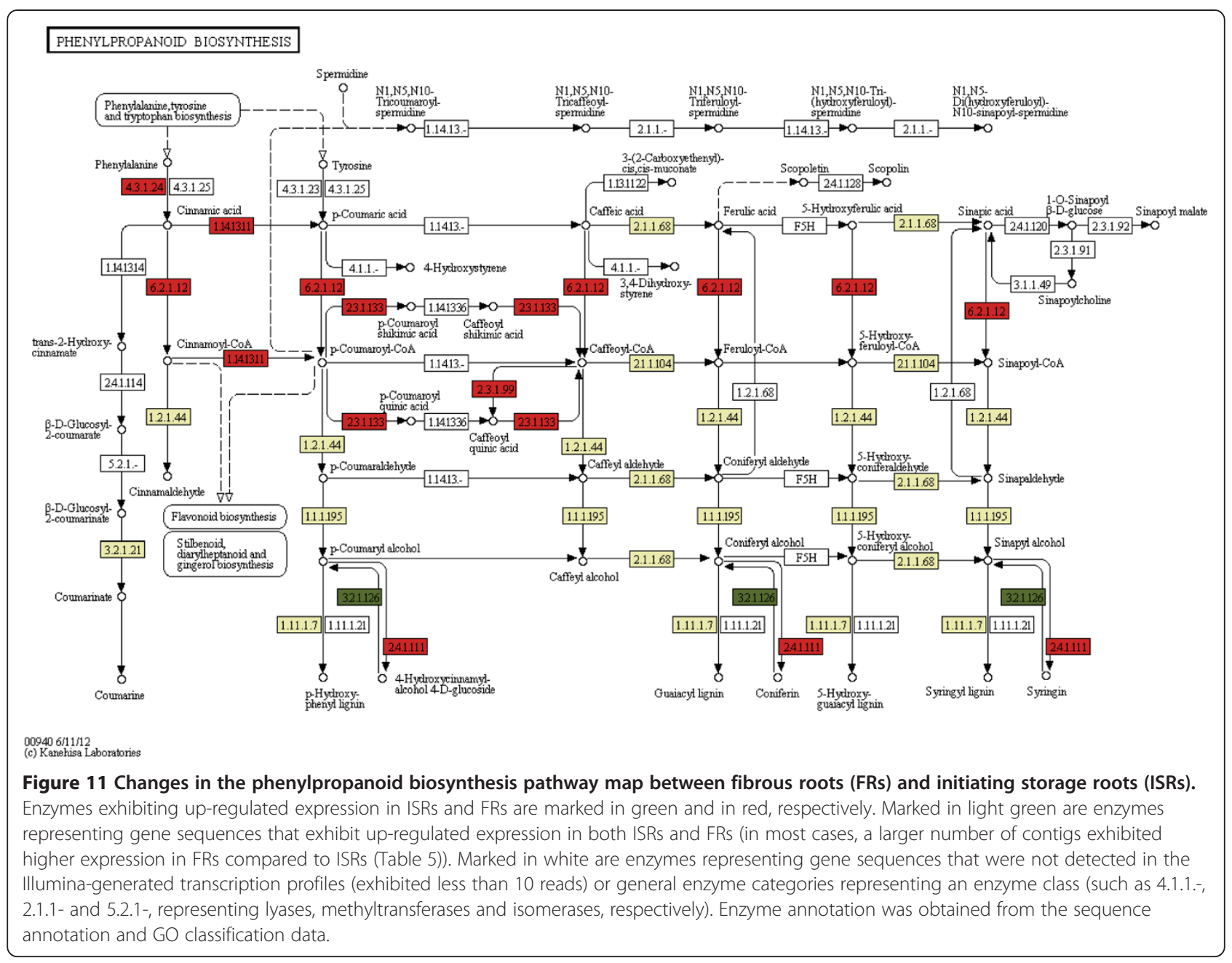

compared to FR (Additional file 5), suggesting reduction in carbon flow toward phenylpropanoid biosynthesis upon SR initiation.

\section{Conclusions}

In the present study, the generation of a database of Georgia Jet sweetpotato root transcriptome, together with a comparison of the expression profiles of ISRs and FRs, enabled the identification of genes involved in the earliest stage of SR formation. Down-regulation in the expression of key genes of the phenylpropanoid biosynthesis pathway upon the change in root fate from FR to a storage organ is indicated, which may be responsible for the significant reduction in lignin levels, representing novel data not previously described in sweetpotato.

The results highlight a reduction in carbon flow toward phenylpropanoid biosynthesis and its delivery into carbohydrate metabolism and starch biosynthesis as major events involved in SR initiation. Specific genes in these pathways were pointed out, providing potential targets for sweetpotato genetic engineering. The results also emphasize the potential importance of delicate control at the level of gene expression of regulators of meristematic tissue identity and maintenance, up-regulation of cell-division regulators and down-regulation of specific GRAS family members, in the SR-initiation process, providing novel data with respect to the specific genes involved. In addition, this study adds a valuable resource of sweetpotato root transcript sequences to the available data, facilitating the identification of genes of interest in this food crop which is among the top seven most important food crops in the world.

\section{Methods}

Plant material and RNA extraction

Georgia Jet is the most important sweetpotato variety grown in Israel. Fifty Georgia Jet plants were produced from transplants and grown in $30-\mathrm{cm}$ pots filled with unfertilized washed sand in a greenhouse at The Volcani Center, Israel, maintained between 22 and $28^{\circ} \mathrm{C}$ with no supplemental light. Roots from 20 plants were sampled 3 to 4 weeks after transplanting as detailed in Villordon et al. 
Table 5 Read count of contigs representing enzymes of the phenylpropanoid biosynthesis pathway in initiating storage roots and fibrous roots

Enzyme/contigs
phenylalanine amn
4.3.1.24)
S_PBL_C14873
S_PBL_C18
S_PBL_C18502
S_PBL_2066
S_PBL_21573
S_PBL_2312

trans-cinnamate 4-monooxygenase (C4H; EC 1.14.13.11)

S_PBL_IrC53776

S_PBL_IrC53138

S_PBL_IrC53079

S_PBL_IrC52110

S_PBL_C98

S_PBL_C2889

S_PBL_C22453

S_PBL_C18141

S_PBL_C17334

4-coumarate-CoA ligase (4CL; EC 6.2.1.12)

S_PBL_C4746

S_PBL_C24867

S_PBL_C22722

S_PBL_C21439

S_PBL_C18044

S_PBL_C158

S_PBL_C1395

S_PBL_C13368

quinate O-hydroxycinnamoyltransferase (EC 2.3.1.99)

S_PBL_C20232

shikimate O-hydroxycinnamoyltransferase (HCT; EC 2.3.1.133)

S_PBL_C8321

S_PBL_c657

S_PBL_c6051

S_PBL_C20232

S_PBL_C20009

S_PBL_C17752

S_PBL_C15462

caffeoyl-CoA O-methyltransferase (CCOAOMT; EC 2.1.1.104)

S_PBL_C6206

S_PBL_C3822

ISR reads FR reads

$\begin{array}{ll}230.1 & 3407.1 \\ 905.5 & 11467.6 \\ 387.3 & 2829.8 \\ 521.0 & 7128.4 \\ 17.8 & 54.5 \\ 344.2 & 3051.1\end{array}$

$\begin{array}{ll}10.3 & 116.5 \\ 262.9 & 1090.4 \\ 345.2 & 6252.8 \\ 434.0 & 4534.9 \\ 391.0 & 9808.5 \\ 285.3 & 1095.8 \\ 154.3 & 2287.8 \\ 707.2 & 2703.6\end{array}$

2703.6

$63.6 \quad 421.2$

$50.5 \quad 162.5$

$70.2 \quad 388.1$

$56.1 \quad 305.7$

$29.9 \quad 379.5$

$119.7 \quad 1904.0$

$52.4 \quad 149.7$

$18.7 \quad 56.7$

8.45

8.490 .9

$185.2 \quad 789.0$

$3.7 \quad 93.0$

$58.0 \quad 346.4$

$8.4 \quad 90.9$

$85.1 \quad 1152.4$

$33.7 \quad 318.6$

$13.1 \quad 43.8$

218.0

645.7

73.9

811.4
Table 5 Read count of contigs representing enzymes of the phenylpropanoid biosynthesis pathway in initiating storage roots and fibrous roots (Continued)

\begin{tabular}{lll}
\hline S_PBL_C2944 & 389.1 & 1204.8 \\
S_PBL_C20480 & 148.7 & 1188.8 \\
S_PBL_C12810 & 89.8 & 916.2 \\
S_PBL_C30794 & 50.5 & 4.3 \\
cinnamoyl-CoA reductase (CCR; EC 1.2.1.44) & & \\
S_PBL_C8128 & 116.9 & 330.3 \\
S_PBL_C5810 & 10499.1 & 30368.4 \\
S_PBL_C2607 & 219.8 & 988.9 \\
S_PBL_C21123 & 86.1 & 495.0 \\
S_PBL_C19132 & 27.1 & 164.6 \\
S_PBL_C10515 & 42.1 & 10.7 \\
caffeate O-methyltransferase & & \\
(COMT; EC 2.1.1.68) & & \\
S_PBL_C978 & 98.2 & 405.2 \\
S_PBL_C538 & 26.2 & 327.1 \\
S_PBL_C20536 & 2.8 & 20.3 \\
S_PBL_C29777 & 78.6 & 15.0
\end{tabular}

cinnamyl-alcohol dehydrogenase

(CAD; EC 1.1.1.195)

\section{S_PBL_IrC54926}

$37.4 \quad 95.1$

S_PBL_IrC54183

S_PBL_IrC53688

S_PBL_IrC53173

S_PBL_IrC52706

S_PBL_IrC52587

S_PBL_IrC26619

S_PBL_C8094

S_PBL_C27372

S_PBL_C22346

S_PBL_C21896

S_PBL_C21084

S_PBL_C18369

S_PBL_C17373

S_PBL_C12615

S_PBL_C20863

S_PBL_C17269

$23.4 \quad 136.8$

$632.3 \quad 8881.6$

$17.8 \quad 96.2$

$599.6 \quad 2211.9$

$355.5 \quad 2451.3$

$6.5 \quad 21.4$

$178.7 \quad 1763.9$

$114.1 \quad 351.7$

$11.2 \quad 49.2$

$27.1 \quad 161.4$

$474.3 \quad 4448.3$

$225.4 \quad 776.1$

$29.0 \quad 139.0$

$328.3 \quad 2762.4$

$186.1 \quad 18.2$

$509.8 \quad 160.4$

beta-glucosidase (EC 3.2.1.21)

S_PBL_c6163

$\begin{array}{ll}4.7 & 13.9 \\ 0.9 & 35.3 \\ 1.9 & 63.1 \\ 39.3 & 101.6 \\ 62.7 & 374.2 \\ 12.2 & 3.2 \\ 64.5 & 2.1\end{array}$


Table 5 Read count of contigs representing enzymes of the phenylpropanoid biosynthesis pathway in initiating storage roots and fibrous roots (Continued)

\begin{tabular}{|c|c|c|}
\hline$\overline{\text { S_PBL_C7311 }}$ & 353.6 & 127.2 \\
\hline S_PBL_C25672 & 55.2 & 8.6 \\
\hline S_PBL_C11889 & 156.2 & 44.9 \\
\hline S_PBL_C50698 & 50.5 & 4.3 \\
\hline S_PBL_C27348 & 74.8 & 23.5 \\
\hline \multicolumn{3}{|c|}{ peroxidase (EC 1.11.1.7) } \\
\hline S_PBL_IrC55044 & 107.6 & 314.3 \\
\hline S_PBL_IrC54488 & 8.4 & 81.2 \\
\hline S_PBL_IrC54403 & 413.5 & 1216.6 \\
\hline S_PBL_IrC54045 & 769.8 & 1999.1 \\
\hline S_PBL_IrC53204 & 427.5 & 1603.6 \\
\hline S_PBL_IrC53085 & 334.9 & 1066.9 \\
\hline S_PBL_IrC52151 & 4.7 & 24.6 \\
\hline S_PBL_C8312 & 44.9 & 1339.5 \\
\hline S_PBL_C750 & 82.3 & 267.3 \\
\hline S_PBL_c6142 & 1.9 & 275.8 \\
\hline S_PBL_C5935 & 2.8 & 23.5 \\
\hline S_PBL_C5844 & 586.5 & 2139.2 \\
\hline S_PBL_C4994 & 61.7 & 609.4 \\
\hline S_PBL_C48762 & 40.2 & 985.7 \\
\hline S_PBL_C44935 & 2.8 & 13.9 \\
\hline S_PBL_C39736 & 11.2 & 40.6 \\
\hline S_PBL_C34320 & 2.8 & 65.2 \\
\hline S_PBL_C29852 & 223.6 & 602.9 \\
\hline S_PBL_C29801 & 24.3 & 215.9 \\
\hline S_PBL_C29652 & 0.9 & 151.8 \\
\hline S_PBL_C2946 & 92.6 & 280.1 \\
\hline S_PBL_C22233 & 5.6 & 22.4 \\
\hline S_PBL_C22191 & 5.6 & 20.3 \\
\hline S_PBL_C2122 & 629.5 & 1831.3 \\
\hline S_PBL_C21120 & 1.9 & 88.7 \\
\hline S_PBL_C18854 & 25.3 & 93.0 \\
\hline S_PBL_C1825 & 22.4 & 182.8 \\
\hline S_PBL_C16766 & 14.0 & 60.9 \\
\hline S_PBL_C1640 & 39.3 & 2253.6 \\
\hline S_PBL_C15871 & 156.2 & 1221.9 \\
\hline S_PBL_C12624 & 42.1 & 143.3 \\
\hline S_PBL_C11598 & 79.5 & 299.3 \\
\hline S_PBL_C11410 & 97.3 & 457.6 \\
\hline S_PBL_C14485 & 116.9 & 32.1 \\
\hline S_PBL_C15642 & 66.4 & 9.6 \\
\hline S_PBL_C7505 & 95.4 & 32.1 \\
\hline S_PBL_C2035 & 1005.6 & 360.3 \\
\hline
\end{tabular}

Table 5 Read count of contigs representing enzymes of the phenylpropanoid biosynthesis pathway in initiating storage roots and fibrous roots (Continued)

\begin{tabular}{lll}
\hline S_PBL_C11972 & 18.7 & 4.3 \\
S_PBL_IrC54799 & 75.8 & 29.9 \\
S_PBL_C4878 & 34.6 & 1.1 \\
S_PBL_C4857 & 549.1 & 97.3 \\
S_PBL_C10185 & 100.1 & 31.0 \\
S_PBL_C17430 & 18.7 & 3.2 \\
S_PBL_IrC54785 & 52.4 & 8.6 \\
Coniferyl-alcohol glucosyltransferase & & \\
(EC 2.4.1.111) & & \\
S_PBL_C1688 & 61.7 & 420.1 \\
S_PBL_C16389 & 61.7 & 1024.2 \\
S_PBL_C13307 & 580.9 & 4191.7 \\
coniferin beta-glucosidase (EC 3.2.1.126) & & \\
S_PBL_C25672 & 55.2 & 8.6 \\
\hline $\begin{array}{l}\text { ISR, Initiating storage root; FR, Fibrous root. Enzyme annotation was obtained } \\
\text { from the sequence annotation and GO classification data. }\end{array}$
\end{tabular}

[7] to identify the timing of SR initiation (marked by initiation of AC cell development). In short, adventitious roots were sectioned at the proximal $3-\mathrm{cm}$ section of the root and the transverse sections were stained with toluidine blue and observed under the microscope. SR initiation was recorded at 4 weeks after transplanting. For each of the remaining 30 plants at this time point, all adventitious roots were sectioned for microscopic analysis and the adjacent root tissue was immediately frozen by plunging into liquid nitrogen. Following microscopic analysis, roots were divided into either ISRs or non-initiated FRs, as shown in Figure 1, and pooled into ISR and FR samples. Root tissue was ground to a fine powder using liquid nitrogen and sea sand (Merck, Darmstadt, Germany), and total RNA was extracted using the Tri reagent (Sigma-Aldrich, Rehovot, Israel). RNA was treated with TURBO DNase (AB Applied Biosystems, Ambion, CA, USA) according to the manufacturer's instructions. The two total RNA samples were examined by capillary electrophoresis using a Shimadzu MultiNA microchip electrophoresis system, and used for the preparation of two types of cDNA libraries as detailed below.

Preparation of a normalized random-primed cDNA library for 454 sequencing

For cDNA synthesis, the two RNA samples were pooled in equal amounts to form a pool designated ISR_FR. A normalized cDNA library was constructed (Eurofins MWG operon, Ebersberg, Germany: http:// www.eurofinsgenomics.eu/). In brief, from the DNasetreated RNA pool, poly(A) + RNA was isolated and used 
for cDNA synthesis. First-strand cDNA synthesis was primed with a N6 randomized primer. Then 454 adapters $A$ and $B$ were ligated to the $5^{\prime}$ and $3^{\prime}$ ends of the cDNA. The cDNA was amplified with 11 PCR cycles using a proofreading enzyme (Additional file 12A, N0). Normalization was carried out by one cycle of denaturation and reassociation of the cDNAs, resulting in N1-cDNAs. Reassociated ds-cDNA was separated from the remaining ss-cDNA (normalized cDNA) by passing the mixture over a hydroxylapatite column. The ss-cDNAs were then amplified with nine PCR cycles (Additional file 12A, N1). For titanium sequencing, the 600- to 800-bp cDNAs were eluted from a preparative agarose gel. Aliquots of the sizefractionated cDNAs were analyzed by capillary electrophoresis (Additional file 12B). The 600- to 800-bp ds-cDNA exhibited the structure described in Additional file 13.

\section{Sequencing by Roche GS FLX technology, using titanium series chemistry}

Following elution from the preparative gel, this size-selected cDNA was sequenced using a Genome Sequencer ${ }^{\mathrm{Tm}}$ (GS) FLX Titanium Instrument (Roche Diagnostics) following a standard protocol [59]. The 454 Life Sciences (Roche Diagnostic) software was used for image and signal processing. A file containing the trace, "base-calling" and quality score data was generated and stored in standard flowgram format (SFF) for subsequent bioinformatic analyses. The sequence data was deposited into the European Nucleotide Archive [EMBL-EBI: PRJEB4145; sample accession ERS255740]. An automated, in-silico-assembly pipeline (Eurofins MWG Operon) was used to assemble the sequence data de novo. FASTA and associated files of 524,607 high-quality, base-called and clipped reads were extracted from the SFF-file and contigs assembled de novo using MIRA (version 2.9.45 × 1; http://chevreux.org/ projects_mira.html; [60]). Mean lengths \pm SD in bases were calculated for particular nucleotide sequence data subsets. MIRA assembly clustered the reads into 55,296 contigs.

\section{Preparation of non-normalized 3'-fragment cDNA libraries from each of the two root samples and Illumina GA-II sequencing}

The extracted total RNA from each of the two pooled root samples-ISRs and non-initiated FRs-was used for cDNA synthesis. The cDNA libraries were prepared by Eurofins MWG Operon. In brief, total RNAs were first sheared with ultrasound ( 10 pulses of $30 \mathrm{~s}$ at $4^{\circ} \mathrm{C}$ ), then poly $(\mathrm{A})+\mathrm{RNA}$ was isolated followed by treatment of the RNA fragments with polynucleotide kinase. An RNA adapter was then ligated to the $5^{\prime}$-phosphate of the 3 'terminal RNA fragments. First-strand cDNA synthesis was performed using an oligo(dT)-adapter primer and MMLVH- reverse transcriptase. The resulting cDNAs were PCR-amplified (number of cycles indicated in
Additional file 14A) using high-fidelity DNA polymerase. Barcode sequences, which were attached to the 5 '-ends of the cDNAs, are described in Additional file 14A. The cDNAs in the size range of 200-450 bp were eluted from preparative agarose gels. Aliquots of the size-fractionated cDNAs were analyzed by capillary electrophoresis (Additional file 14B). The resulting, ds-cDNAs were of about 200-450 bp as demonstrated in Additional file 14C and contained adapter sequences (Additional file 14D). Illumina sequencing using the GA-II platform was performed by Eurofins MWG operon according to the manufacturer's instructions (Illumina, San Diego, CA, USA). A quality score of Q30 was used [Q30 being associated with an error rate (namely the probability that a base is called erroneously) of $0.1 \%]$. The specifications concerning Q30 were as follows: $\geq 70 \%$ of the reads having Q30 for reads longer than 75 bases and $\geq 75 \%$ of the reads having Q30 for reads up to 50 bases.

\section{Illumina read mapping against the 454-generated sweetpotato root transcript sequences}

The raw Illumina reads were sorted using their tags (ISR: GAGT and FR: CTTG; Additional file 14A), resulting in the following number of reads per sample (HiSeq 2000, $1 \times 100 \mathrm{bp}$ ): 14,780,229 and 17,703,982 for ISR and FR, respectively. The sequence data was deposited into the European Nucleotide Archive [EMBL-EBI: PRJEB4145; sample accession numbers ERS255744 and ERS255745 for ISRs and FRs, respectively]. Prior to the read mapping, the four tag bases were removed from its $5^{\prime}$ end. These read sequences were then used as input for read mapping against the set of 55,296 FLX EST contigs. The mapping was conducted using the software BWA 0.5.8c (http://bio-bwa.sourceforge.net). Post-processing of the mapping was conducted with samtools $0.1 .12 \mathrm{a}$ (http://samtools.sourceforge.net). The total number of mapped reads for each of the two samples (ISR and FR) was quantified. To enable a direct comparison between the two samples, the read count per EST contig was normalized using DESeq [61].

Validation of the Illumina-generated transcription profiles of FR and ISR samples using real-time qRT-PCR analyses RNA samples were prepared from either FRs or ISRs as described above, using at least four biological replicates. Each replicate contained root tissue derived from 30 plants. RNA samples were cleaned of DNA contamination using RQ1 RNase-free DNase (Promega WI, USA). cDNA was synthesized using $1 \mu \mathrm{g}$ of total RNA and Superscript II (Invitrogen) reverse transcriptase, according to the manufacturer's instructions. First-strand cDNA was used for qRT-PCR analyses. Primer3web version 4.0: http:// primer3.ut.ee) was used for primer design, and qRTPCR was performed on the ROTOR-GENE ${ }^{\text {тм }} 6000$ 
(Corbett LIFE SCIENCE, Qiagen), using SYBR ${ }^{\circ}$ Green. A total reaction volume of $15 \mu \mathrm{l}$ was used. The reaction mix included $3 \mu \mathrm{l}$ template, $0.3 \mu \mathrm{l}$ reverse primer, $0.3 \mu \mathrm{l}$ forward primer, $7.5 \mu \mathrm{l}$ Absolute Blue QPCR SYBR Green ROX Mix (Thermo Fisher Scientific Inc. MA, USA), and $3.9 \mu \mathrm{l}$ RNA-free water. A qRT-PCR assay was performed using the following conditions: $95^{\circ} \mathrm{C}$ for 9 min followed by 40 cycles of $95^{\circ} \mathrm{C}$ for $10 \mathrm{~s}, 60^{\circ} \mathrm{C}$ for $10 \mathrm{~s}$ and $72^{\circ} \mathrm{C}$ for $20 \mathrm{~s}$. The $2^{-\Delta \Delta C T}$ method [62] was used to normalize and calibrate transcript values relative to the $18 \mathrm{~S}$ ribosomal protein, whose expression did not change across sweetpotato root types or developmental stages. The FR sample was used as the calibrator sample. Primer sequences were designed according to the respective contigs assembled from reads that were obtained from the 454-sequencing results and are described in Additional file 15. Use of the oligonucleotide primers listed in Additional file 15 resulted in approximately equal efficiencies of amplification for the various target and reference genes. Each set of experiments was repeated at least four times and final relative quantification results are given as average \pm SE.

\section{Functional annotation and analyses of GO-term and KEGG-pathway enrichment}

The assembled transcripts were used to query public genomic databases (i.e. NCBI Nr) using BLASTX (Eurofins MWG Operon) and annotations of the two best hits for each contig were recorded. BlastoGO [22] was used to obtain GO annotations and Ontologizer [23] was used to perform GO functional classification. The contigs were further classified using GOSlim (http://www.geneontology. org/GO.slims.shtml). To assign the detected contigs to biological pathways, sequences were compared using BLASTX with an E-value cutoff of $<10 \mathrm{E}-3$ against the KEGG database (http://www.genome.jp/kegg/). The differentially expressed contigs in ISRs and FRs were analyzed for GO-category enrichment relative to the root transcriptome database using AgriGO [44]. The differentially expressed contigs in ISRs and FRs were analyzed for KEGG-pathway enrichment relative to the rest of the root transcriptome database using Fisher's Exact Test and FDR correction [48].

\section{Starch content analysis}

Root samples of Georgia Jet were pooled from five to seven plants at 1,2, 3 and 4 weeks after transplanting, spanning the period of SR initiation [7]. The tissue was ground in liquid nitrogen using a mortar and pestle and ethanol-suspended root samples were extracted three times in hot $80 \%(\mathrm{v} / \mathrm{v})$ ethanol. The insoluble residue that remained after ethanolic extraction was resuspended in $30 \mathrm{mM} \mathrm{HCl}$ and boiled for $30 \mathrm{~min}$. After cooling, the $\mathrm{pH}$ was adjusted to 4.5 with $\mathrm{KOH}$. The gelatinized starch was digested for $60 \mathrm{~min}$ at $50^{\circ} \mathrm{C}$ with approximately $36 \mathrm{U}$ of amyloglucosidase from Aspergillus oryza [63]. Reducing sugars were determined according to Miller [64]. Three biological replicates were used for each analysis.

\section{Additional files}

\begin{abstract}
Additional file 1: Annotations of the two best hits for each contig (E value $\leq 1.0 \mathrm{E}-3$ ). Annotations are listed in boxes $12-161,668$.

Additional file 2: Species distribution of the BLAST results. Presented data were extracted from the detailed data of the number of BLAST results matching each plant species. For each contig, the best BLAST result was used.
\end{abstract}

Additional file 3: Functional annotations of sweetpotato root transcripts (combined initiating storage and fibrous root samples) using GOSlim. The three GO functional categories (BP, MF, CC) were segregated into separate worksheets. Whenever relevant, contigs were assigned to more than one GO term.

Additional file 4: Functional classification of sweetpotato root transcripts (combined initiating storage and fibrous root samples) to biological pathways using KEGG.

Additional file 5: Total normalized read counts in the fibrous root and initiating storage root samples. Illumina GA-Ilx technology was carried out to compare the transcription profiles of a pooled sample of fibrous roots and a pooled sample of initiating storage roots and reads were mapped against our database of 55,296 FLX contigs. Normalization was performed as described in 'Methods'. One annotation is given for each contig. Annotations of the two best hits for each contig (E value $\leq 1.0 \mathrm{E}-3$ ) are available in Additional file 1.

Additional file 6: Differentially expressed contigs between initiating storage roots (ISRs) and fibrous roots (FRs). A contig was considered as differentially expressed if two conditions were met: (1) at least 10 reads were mapped to this contig in at least one of the samples and (2) no reads were mapped to this contig in the other sample, or the foldchange between its read number in each sample was at least 2.5 . The up-regulated contigs in ISR and FR are segregated into separate worksheets. Fold-change expression was calculated relative to the read number in the ISR sample. One annotation is given for each contig. Annotations of the two best hits for each contig ( $E$ value $\leq 1.0 \mathrm{E}-3$ ) are available in Additional file 1.

Additional file 7: GO-term enrichment in the initiating storage root sample relative to the root transcriptome database. The specific contigs for each $\mathrm{GO}$ term in categories biological process (BP), molecular function (MF) and cellular component (CC) are given on separate sheets. ISR - initiating storage root sample.

Additional file 8: Tree representation of enriched GO terms in the initiating storage root sample. A more intense color indicates a higher number of contigs. ISR - initiating storage root sample.

Additional file 9: GO-term enrichment in the fibrous root sample relative to the root transcriptome database. The specific contigs for each GO term in categories biological process (BP), molecular function (MF) and cellular component (CC) are given on separate sheets. FR fibrous root sample.

Additional file 10: Tree representation of enriched GO terms in the fibrous root sample. A more intense color indicates a higher number of contigs. FR - fibrous root sample.

Additional file 11: Read number of contigs representing the initiating storage root-enriched sulfur metabolism in the KEGG pathway.

Additional file 12: Analysis of the PCR-amplified CDNAs on a Shimadzu MultiNA microchip electrophoresis system. A. Analysis of the PCR-amplified N0 and N1 CDNAs. B. Analysis of the size-fractionated N1 cDNAs. $\mathrm{M}-100$ bp ladder. 
Additional file 13: Description of the CDNA used for 454 sequencing. The 454 adapter sequences are underlined. The first four bases of adapter primers $\mathrm{A} 1$ and $\mathrm{B} 1$ represent phosphorothioate-modified bases as specified by Roche. In addition, primer B1 is 5'-biotinylated. The barcode sequence is: CACACG.

Additional file 14: Properties of the non-normalized CDNA samples. A. PCR amplification of the CDNA samples. B. Analysis of the PCR-amplified 3'-fragment CDNAs on the Shimadzu MultiNA microchip electrophoresis system. C. Analysis of the size-fractionated cDNAs on the Shimadzu MultiNA microchip electrophoresis system. D. Description of CDNA for Illumina sequencing. Illumina adapter sequences are underlined. M - 100 bp ladder; ISR - initiating storage root sample; FR - fibrous root sample.

Additional file 15: List of oligonucleotide primer sequences used for the quantitative RT-PCR analyses.

\section{Abbreviations}

AC: Anomalous cambium; DAT: Days after transplanting; FR: Fibrous root; ISR: Initiating storage root; LC: Lignified cell; NGS: Next-generation sequencing; RVC: Regular vascular cambium; SR: Storage root.

\section{Competing interests}

The authors declare that they have no competing interests.

\section{Authors' contributions}

The study was conceived by NF, DL and AV. NF drafted the manuscript. YK designed the experimental setup, together with NF, DL and $A V$, and performed the experiments, including tissue sections, RNA extractions and starch content analyses. EL performed the quantitative RT-PCR analyses. JS contributed to the experiment performance and data analysis. TSP performed the bioinformatics analyses, including functional annotation of the database. ADF participated in the bioinformatics analyses, including GO category and KEGG pathway enrichment. AH generated the sweetpotato transcriptome web page. L Althan contributed to the plant material preparation and maintenance. L Adani Nadir participated in RNA extractions. All authors read and approved the final manuscript.

\section{Acknowledgements}

Support for this research was provided by a grant from The USA-Israel Binational Agricultural Research and Development grant number US-4015-07.

\section{Author details}

${ }^{1}$ Institute of Plant Sciences, The Volcani Center, Agricultural Research Organization, Bet Dagan 50250, Israel. '2LS AgCenter School of Plant, Environmental, and Soil Sciences, 137 J.C. Miller Hall, Baton Rouge, LA 70803-2120, USA. ${ }^{3}$ LSU AgCenter Sweet Potato Research Station, Chase, LA 71324, USA. ${ }^{4}$ Medical Goldyne Savad Institute of Gene Therapy, Hadassah Medical Center, Jerusalem 91120, Israel. ${ }^{5}$ Agricultural Engineering, Sensing, Information and Mechanization Engineering, The Volcani Center, Agricultural Research Organization, Bet Dagan 50250, Israel.

\section{Received: 1 January 2013 Accepted: 19 June 2013}

Published: 9 July 2013

\section{References}

1. FAOSTAT Data. [http://faostat3.fao.org/home/index.html].

2. Woolfe J: Sweetpotato, an Untapped Food Source. Cambridge: Cambridge University Press; 1992.

3. The Nutrition Action Health Letter from the Center for Science in the Public Interest. USA [http://www.cspinet.org/nah/].

4. Togari Y: A study of tuberous root formation in sweet potato. Bull Natl Agric Exp Statn Tokyo 1950, 68:1-96.

5. Wilson LA, Lowe SB: The anatomy of the root system in West Indian sweet potato [lpomoea batatas (L.) Lam.] cultivars. Ann Bot (Lond) 1973, 37:633-643

6. Belehu T, Hammes PS, Robbertse PJ: The origin and structure of adventitious roots in sweetpotato (Ipomoea batatas). Aust J Bot 2004, 52:551-558.

7. Villordon $A Q$, La Bonte DR, Firon N, Kfir Y, Schwartz A, Pressman E: Characterization of adventitious root development in sweetpotato. Hortscience 2009, 44:651-655.
8. You MK, Hur CG, Ahn YS, Suh MC, Jeong BC, Shin JS, Bae JM: Identification of genes possibly related to storage root induction in sweetpotato. FEBS Lett 2003, 53:101-105

9. McGregor C: Differential expression and detection of transcripts in sweetpotato (Ipomoea batatas (L.) LAM.) using CDNA microarrays. PhD thesis. Louisiana State University and Agricultural and Mechanichal College; 2006. http://etd. Isu.edu/docs/available/etd-05092006-145816/.

10. Kim SH, Mizuno K, Fujimura K: Isolation of MADS-box genes from sweet potato (Ipomoea batatas (L.) Lam.) expressed specifically in vegetative tissues. Plant Cell Physiol 2002, 43:314-322.

11. Kim SH, Hamada T, Otani M, Shimada T: Isolation and characterization of MADS box genes possibly related to root development in sweetpotato (Ipomoea batatas L. Lam.). J Plant Biol 2005, 48:387-393.

12. Kim SH, Hamada T, Otani M, Shimada T: Cloning and characterization of sweetpotato MADS-box gene (IbAGL17) isolated from tuberous root. Plant Biotechnol 2005, 22:217-220.

13. Lalusin AG, Nishita K, Kim S-H, Ohta M, Fujimura T: A new MADS-box gene (IbMADS10) from sweet potato (Ipomoea batatas (L.) Lam) is involved in the accumulation of anthocyanin. Mol Genet Genom 2006, 275:44-54.

14. Ku AT, Huang YS, Wang YS, Ma D, Yeh KW: IbMADS1 (Ipomoea batatas MADS-box 1 gene) is involved in tuberous root initiation in sweet potato (Ipomoea batatas). Ann Bot 2008, 102:57-67.

15. Noh SA, Lee HS, Huh EJ, Huh GH, Paek KH, Shin JS, Bae JM: SRD1 is involved in the auxin-mediated initial thickening growth of storage root by enhancing proliferation of metaxylem and cambium cells in sweetpotato (Ipomoea batatas). J Exp Bot 2010, 61:1337-1349.

16. Tanaka M, Takahata Y, Nakatani M: Analysis of genes developmentally regulated during storage root formation of sweetpotato. J Plant Physiol 2005, 162:91-102.

17. Tanaka M, Kato N, Nakayama H, Nakatani M, Takahata Y: Expression of class 1 Knotted1-like homeobox genes in the storage roots of sweetpotato (Ipomoea batatas). J Plant Physiol 2008, 165:1726-1735.

18. Schafleitner R, Tincopa LR, Palomino O, Rossel G, Robles RF, Alagon R, Rivera C, Quispe C, Rojas L, Pacheco JA, Solis J, Cerna D, Kim JY, Hou J, Simon R: A sweetpotato gene index established by de novo assembly of pyrosequencing and Sanger sequences and mining for gene-based microsatellite markers. BMC Genomics 2010, 11:604.

19. Wang ZY, Fang BP, Chen JY, Zhang XJ, Luo ZX, Huang L, Chen X, Li Y De novo assembly and characterization of root transcriptome using Illumina paired-end sequencing and development of CSSR markers in sweetpotato (Ipomoea batatas). BMC Genomics 2010, 11:726.

20. Tao X, Gu Y-H, Wang H-Y, Zheng W, Li X, Zhao C-W, Zhang YZ: Digital gene expression analysis based on integrated de novo transcriptome assembly of sweetpotato [lpomoea batatas (L.) Lam.]. PLoS One 2012, 7:e36234.

21. Xie F, Burklew CE, Yang Y, Liu M, Xiao P, Zhang B, Qiu D: De novo sequencing and comprehensive analysis of purple sweet potato (Ipomoea batatas L.) transcriptome. Planta 2012, 236:101-113.

22. Conesa A, Gotz S, Garcia-Gomez JM, Terol J, Talon M, Robles M: Blast2GO: a universal tool for annotation, visualization and analysis in functional genomics research. Bioinformatics 2005, 21:3674-3676.

23. Bauer S, Grossmann S, Vingron M, Robinson PN: Ontologizer 2.0 - A multifunctional tool for $\mathrm{GO}$ term enrichment analysis and data exploration. Bioinformatics 2008, 24:1650-1651.

24. GO Slim and Subset Guide. [http://www.geneontology.org/GO.slims.shtml].

25. Kyndt T, Denil S, Haegeman A, Trooskens G, De Meyer T, Van Criekinge W, Gheysen G: Transcriptome analysis of rice mature root tissue and root tips in early development by massive parallel sequencing. J Exp Bot 2012, 63:2141-2157.

26. Yang $H, H u L$, Hurek T, Reinhold-Hurek B: Global characterization of the root transcriptome of a wild species of rice, Oryza longistaminata, by deep sequencing. BMC Genomics 2010, 11:705.

27. Swarbreck SM, Lindquist EA, Ackerly DD, Andersen GL: Analysis of leaf and root transcriptomes of soil-grown Avena barbata plants. Plant Cell Physiol 2011, 52:317-332.

28. Maeshima M, Sasaki T, Asahi T: Characterization of major proteins in sweetpotato tuberous roots. Phytochemistry 1985, 24:1899-1902.

29. Nakamura K, Ohto M, Yoshida N, Nakamura K: Sucrose induced accumulation of beta-amylase occurs concomitant with the accumulation of starch and sporamin in leaf-petiole cuttings of sweet potato. Plant Physiol 1991, 96:902-909. 
30. Lee DK, Ahn JH, Song SK, Choi YD, Lee JS: Expression of an expansin gene is correlated with root elongation in soybean. Plant Physio/ 2003, 131:985-997.

31. Noh SA, Lee HS, Kim YS, Paek KH, Shin JS, Bae JM: Down-regulation of the IbEXP1 gene enhanced storage root development in sweetpotato. J Exp Bot 2012, 64:129-142.

32. Tiessen A, Hendriks JHM, Stitt M, Branscheid A, Gibon Y, Farré EM, Geigenberger P: Starch synthesis in potato tubers is regulated by post-translational redox modification of ADP-glucose pyrophosphorylase. A novel regulatory mechanism linking starch synthesis to the sucrose supply. Plant Cell 2002, 14:2191-2213.

33. Osnato M, Stile MR, Wang Y, Meynard D, Curiale S, Guiderdoni E, Liu Y, Horner DS, Ouwerkerk PBF, Pozzi C, Mueller K, Salamini F, Laura Rossini L: Cross talk between the KNOX and ethylene pathways is mediated by intron-binding transcription factors in barley. Plant Physiol 2010, 154:1616-1632.

34. Agusti J, Lichtenberger R, Schwarz M, Nehlin L, Greb T: Characterization of transcriptome remodeling during cambium formation identifies MOL1 and RUL1 as opposing regulators of secondary growth. PLOS Genet 2011 7(2):e1001312. doi:10.1371/journal.pgen.1001312.

35. Schrader J, Nilsson J, Mellerowicz E, Berglund A, Nilsson P, Hertzberg M, Sandberga G: A high-resolution transcript profile across the wood-forming meristem of poplar identifies potential regulators of cambial stem cell identity. Plant Cell 2004, 16:2278-2292.

36. Ravi V, Naskar SK, Makeshkumar T, Babu B, Krishnan BSP: Molecular physiology of storage root formation and development in sweet potato (Ipomoea batatas (L.) Lam.). J Root Crops 2009, 35:1-27.

37. Mele G, Ori N, Sato Y, Hake S: The knotted1-like homeobox gene BREVIPEDICELLUS regulates cell differentiation by modulating metabolic pathways. Genes Dev 2013, 17:2088-2093.

38. Yuan J, Chen D, Ren Y, Zhang X, Zhao J: Characteristic and expression analysis of a metallothionein gene, OsMT2b, down-regulated by cytokinin suggests functions in root development and seed embryo germination of rice. Plant Physiol 2008, 146:1637-1650.

39. Van Loon LC, Van Strien EA: The families of pathogenesis-related proteins, their activities, and comparative analysis of PR-1 type proteins. Physiol Mol Plant Pathol 1999, 55:85-97.

40. Chen HJ, Su CT, Lin CH, Huang GJ, Lin YH: Expression of sweet potato cysteine protease SPCP2 altered developmental characteristics and stress responses in transgenic Arabidopsis plants. J Plant Physiol 2010, 167:838-847.

41. Vanholme R, Morreel K, Ralph J, Boerjan W: Lignin engineering. Curr Opin Plant Biol 2008, 11:278-285

42. Hatfield $R$, Vermerris $W$ : Lignin formation in plants. The dilemma of linkage specificity. Plant Physiol 2001, 126:1351-1357.

43. Testone G, Condello E, Verde I, Nicolodi C, Caboni E, Dettori MT, Vendramin E, Bruno L, Bitonti MB, Mele G, Giannino D: The peach (Prunus persica L. Batsch) genome harbours 10 KNOX genes, which are differentially expressed in stem development, and the class 1 KNOPE1 regulates elongation and lignification during primary growth. J Exp Bot 2012, 63:5417-5435.

44. Du Z, Zhou X, Ling Y, Zhang Z, Su Z: AgriGO: a GO analysis toolkit for the agricultural community. Nucleic Acids Res 2010, 38:W64-W70.

45. Carles CC, Choffnes-Inada D, Reville K, Lertpiriyapong K, Fletcher JC: ULTRAPETALA1 encodes a SAND domain putative transcriptional regulator that controls shoot and floral meristem activity in Arabidopsis. Development 2005, 132:897-911.

46. Carles CC, Lertpiriyapong K, Reville K, Fletcher JC: The ULTRAPETALA1 gene functions early in Arabidopsis development to restrict shoot apical meristem activity, and acts through WUSCHEL to regulate floral meristem determinacy. Genetics 2004, 167:1893-1903.

47. Zhao C, Craig JC, Petzold HE, Dickerman AW, Beers EP: The xylem and phloem transcriptomes from secondary tissues of the Arabidopsis root-hypocotyl. Plant Physiol 2005, 138:803-818.

48. Benjamini $Y$, Hochberg $Y$ : Controlling the false discovery rate: a practical and powerful approach to multiple testing. J Royal Stat Soc 1995, 57:289-300.

49. Sojikul $P$, Kongsawadworakul P, Viboonjun U, Thaiprasit J, Intawong B, Narangajavana J, Svasti MR: AFLP-based transcript profiling for cassava genome-wide expression analysis in the onset of storage root formation. Physiol Plant 2010, 140:189-198.
50. Khan MS, Haas FH, Samami AA, Gholami AM, Bauer A, Fellenberg K, Reichelt M, Hänsch R, Mendel RR, Meyer AJ, Wirtz M, Hell R: Sulfite reductase defines a newly discovered bottleneck for assimilatory sulfate reduction and is essential for growth and development in Arabidopsis thaliana. Plant Cell 2010, 22:1216-1231.

51. Yang J, An D, Zhang P: Expression profiling of cassava storage roots reveals an active process of glycolysis/gluconeogenesis. J Integr Plant Biol 2011, 53:193-211.

52. Schilmiller AL, Stout J, Weng JK, Humphreys J, Ruegger MO, Chapple C: Mutations in the cinnamate 4-hydroxylase gene impact metabolism, growth and development in Arabidopsis. Plant J 2009, 60:771-782.

53. Raes J, Rohde A, Christensen JH, Van de Peer Y, Boerjan W: Genome-wide characterization of the lignification toolbox in Arabidopsis. Plant Physiol 2003, 133:1051-1071

54. Kajita S, Katayama Y, Omori S: Alterations in the biosynthesis of lignin in transgenic plants with chimeric genes for 4-coumarate:coenzyme A ligase. Plant Cell Physiol 1996, 37:957-965.

55. Kajita S, Hishiyama S, Tomimura Y, Katayama Y, Omori S: Structural characterization of modified lignin in transgenic tobacco plants in which the activity of 4-coumarate:coenzyme A ligase is depressed. Plant Physiol 1997, 114:871-879.

56. Lee D, Meyer K, Chapple C, Douglas CJ: Antisense suppression of 4-coumarate: coenzyme A ligase activity in Arabidopsis leads to altered lignin subunit composition. Plant Cell 1997, 9:1985-1998.

57. Hu WJ, Harding SA, Lung J, Popko JL, Ralph J, Stokke DD, Tsai CJ, Chiang VL: Repression of lignin biosynthesis promotes cellulose accumulation and growth in transgenic trees. Nat Biotechnol 1999, 17:808-812.

58. Aharoni A, Galili G: Metabolic engineering of the plant primary-secondary metabolism interface. Curr Opin Biotechnol 2011, 22:239-244.

59. Margulies M, Egholm M, Altman WE, Attiya S, Bader JS, Bemben LA, Berka J, Braverman MS, Chen YJ, Chen Z, Dewell SB, Du L, Fierro JM, Gomes XV, Godwin BC, He W, Helgesen S, Ho CH, Irzyk GP, Jando SC, Alenquer MLI, Jarvie TP, Jirage KB, Kim J-B, Knight JR, Lanza JR, Leamon JH, Lefkowitz SM, Lei M, Li J: Genome sequencing in microfabricated high-density picolitre reactors. Nature 2005, 437:376-380.

60. Chevreux B, Wetter T, Suhai S: Genome sequence assembly using trace signals and additional sequence information. In Comput Sci Biol: Proc. German Conference on Bioinformatics GCB'99 GCB. ; 1990:45-56.

61. Anders $S$, Huber W: Differential expression analysis for sequence count data. Genome Biol 2010, 11:R106.

62. Livak KJ, Schmittgen TD: Analysis of relative gene expression data using real-time quantitative PCR and the 2-[delta][delta]CT method. Methods 2001, 25:402-408.

63. Hubbard NL, Pharr DM, Huber SC: Role of sucrose phosphate synthase in sucrose biosynthesis in ripening bananas and its relationship to the respiratory climacteric. Plant Physiol 1990, 94:201-208.

64. Miller GL: Use of dinitrosalicylic acid reagent for determination of reducing sugars. Anal Chem 1959, 31:426-428.

doi:10.1186/1471-2164-14-460

Cite this article as: Firon et al:: Transcriptional profiling of sweetpotato (Ipomoea batatas) roots indicates down-regulation of lignin biosynthesis and up-regulation of starch biosynthesis at an early stage of storage root formation. BMC Genomics 2013 14:460.

\section{Submit your next manuscript to BioMed Central and take full advantage of:}

- Convenient online submission

- Thorough peer review

- No space constraints or color figure charges

- Immediate publication on acceptance

- Inclusion in PubMed, CAS, Scopus and Google Scholar

- Research which is freely available for redistribution 